\title{
Ray-tracing and Fokker-Planck modelling of the effect of plasma current on the propagation and absorption of lower hybrid waves
}

\author{
F Imbeaux and Y Peysson \\ Association EURATOM-CEA, CEA/DSM/DRFC, CEA Cadarache, 13108 Saint Paul lez \\ Durance, France
}

Received 23 March 2005, in final form 29 July 2005

Published 21 October 2005

Online at stacks.iop.org/PPCF/47/2041

\begin{abstract}
Waves at a frequency close to the lower hybrid (LH) resonance are widely used in tokamaks for non-inductive current drive. Modelling of LH waves is usually carried out by combining a Ray-tracing (RT) code for computing the LH waves propagation to a solver of the Fokker-Planck (FP) equation which calculates an electron distribution function self-consistently with the waves absorption. The DELPHINE code has been developed along this approach with accurate treatment of the magnetic equilibrium and the fast electrons dynamics in momentum space. Using this code, the influence of the plasma current on the LH waves propagation and absorption is investigated in detail. High plasma current is found to broaden the absorbed LH spectrum towards high phase velocities, thus increasing the current drive efficiency of the waves. The shape of the current density profile also has an impact on the propagation of the waves and the resulting power deposition. In discharges where the current profile is dominated by LH current drive (LHCD), this dependence leads to the auto-regulation of the LHCD via the current density profile. The RT/FP technique reproduces at least qualitatively some of the experimental trends, though inconsistencies still remain. Perspectives for improving the relevance of the modelling are discussed.
\end{abstract}

(Some figures in this article are in colour only in the electronic version)

\section{Introduction}

Waves at a frequency close to the lower hybrid ( $\mathrm{LH})$ resonance are widely used in tokamaks for non-inductive current drive. Using these waves, steady-state plasma discharges could be sustained for durations well beyond the current diffusion time [1,2]. In the JET tokamak, LH waves are used to preform the current profile in order to trigger internal transport barriers and also to sustain them during the main heating phase $[3,4]$. Regimes with internal transport 
barriers at zero loop voltage have also been obtained in discharges with full LH current drive (LHCD) [5,6]. In a next step tokamak, LH waves appear as the only efficient method for driving current far off-axis, providing access to advanced tokamak regimes [7].

Modelling of $\mathrm{LH}$ waves has also been extensively carried out for more than twenty years [8-15]. A presently widely used method is to combine a Ray-tracing (RT) code for computing the LH waves propagation to a solver of the Fokker-Planck (FP) equation which calculates an electron distribution function self-consistently with the waves absorption. Recent efforts have been carried out to provide more accurate predictions within the RT and quasilinear approximations. For the RT codes, this leads to take into account accurately the plasma geometry, i.e. using two-dimensional (2D) Grad-Shafranov magnetic equilibrium and also including the effect of the toroidal magnetic field ripple because of the discrete number of toroidal magnetic field coils [15]. For FP codes, this means describing the electron distribution function in a 2D momentum space (in both parallel and perpendicular directions to the magnetic field), including the effect of magnetic trapping, with fully relativistic equations. These efforts lead to the code named DELPHINE, which is presented here.

As an example of the application of the DELPHINE code, the role of plasma current density profile on LH waves propagation and absorption is investigated in detail. This parameter is indeed critical for LH waves dynamics. First, it has an influence on the propagation, since the dispersion relation involves the local magnetic field value. Using a numerical mapping of the electrostatic cold plasma dispersion relation, it has been shown that high plasma current and high magnetic shear tend to increase the rate of the toroidal $n_{\|}$-upshift [16], $n_{\|}$being the refractive index of the LH wave vector along the magnetic field. These effects are still present in the full electromagnetic dispersion relation including hot plasma effects that are considered in DELPHINE. Moreover, the plasma current also plays a crucial role in the definition of the parallel refractive index domain in which the waves can propagate, in particular its low boundary, and so determines the maximum velocity of resonant electrons. This has a direct impact on the current drive efficiency of the waves, which increases with this upper resonant velocity according to the well-known Fisch-Boozer current drive theory [17]. Experimental observations of the effect of the total plasma current on LH waves power deposition and current drive efficiency have been reported in $[15,18-$ 20]. The LH power deposition profile, measured by fast electron bremsstrahlung (FEB) tomography [21], becomes broader at higher current, an effect which has also been observed in JET [22] and FTU [23]. Moreover the current drive efficiency, determined at zero loop voltage, is found to increase as a function of the plasma current [18-20]. In this work, we interprete these phenomena using the RT/FP code DELPHINE. The underlying physics of this tool is described in section 2. In section 3, the influence of the plasma current on the current drive efficiency is investigated, keeping the shape of the equilibrium current density profile constant. The behaviour of the rays is investigated in detail, in order to bring to light some of the fundamental mechanisms of propagation as modelled by RT. The simulation results are then discussed and compared with the experiments reported in [18]. In addition, RT/FP modelling also predicts a strong dependence of LH waves propagation and absorption on the shape of the current density profile, as reported in [16]. This dependence is described in section 4. It has important consequences for the predictive modelling of scenarios with dominant LHCD, which are discussed in section 5: it introduces a feedback loop between the LH waves driven current and the plasma current density, which complicates the modelling of such discharges. Finally, in section 6, the predictions of RT/FP modelling about this dependence on plasma current density is compared with experimental data. This leads to some considerations on the present capability of RT/FP codes to model the LH waves propagation and absorption. 


\section{Presentation of the code}

The code DELPHINE includes an RT module, which calculates the LH waves propagation and the absorption along the rays for a given electron distribution function. In order to take quasilinear effects into account, the RT part is coupled to an FP solver, which calculates the deformation of the distribution function in momentum space due to a given quasilinear diffusion coefficient (calculated by the module which computes the absorption along the rays). Following the usual method, the absorption and FP modules are iterated successively until they have converged towards consistent power deposition and electron distribution function.

The main features of the RT are the following: the waves dispersion relation equation is solved in the LH frequency approximation, including thermal corrections to the cold plasma dielectric tensor with the assumption of strongly magnetized electrons and weakly magnetized ions [9]: i.e.

$$
D(\vec{r}, \vec{k}, \omega)=P_{6} n_{\perp}^{6}+P_{4} n_{\perp}^{4}+P_{2} n_{\perp}^{2}+P_{0}=0
$$

with

$$
\begin{aligned}
& P_{0}=\varepsilon_{\|}\left[\left(n_{\|}^{2}-\varepsilon_{\perp}\right)^{2}-\varepsilon_{x y}^{2}\right], \\
& P_{2}=\left(\varepsilon_{\|}+\varepsilon_{\perp}\right)\left(n_{\|}^{2}-\varepsilon_{\perp}\right)+\varepsilon_{x y}^{2}, \\
& P_{4}=\varepsilon_{\perp}, \\
& P_{6}=-\left(\frac{3}{2} \frac{\omega_{\mathrm{pi}}^{2}}{\omega^{2}} \frac{v_{\mathrm{i}}^{2}}{c^{2}}+\frac{3}{8} \frac{\omega_{\mathrm{pe}}^{2}}{\Omega_{c e}^{2}} \frac{\omega^{2}}{\Omega_{c e}^{2}} \frac{v_{\mathrm{e}}^{2}}{c^{2}}\right), \\
& \varepsilon_{\perp}=1+\frac{\omega_{\mathrm{pe}}^{2}}{\Omega_{c e}^{2}}-\frac{\omega_{\mathrm{pi}}^{2}}{\omega^{2}}, \\
& \varepsilon_{\|}=1-\frac{\omega_{\mathrm{pe}}^{2}}{\omega^{2}}-\frac{\omega_{\mathrm{pi}}^{2}}{\omega^{2}}, \\
& \varepsilon_{x y}=\frac{\omega_{\mathrm{pe}}^{2}}{\omega \Omega_{c e}}, \\
& \Omega_{c e}=\frac{e B}{m_{\mathrm{e}}}, \\
& \omega_{\mathrm{pe}}=\sqrt{\frac{n_{\mathrm{e}} e^{2}}{\varepsilon_{0} m_{\mathrm{e}}}}, \\
& \omega_{\mathrm{pi}}^{2}=\sum_{i=\mathrm{species}} \frac{n_{\mathrm{i}} Z_{\mathrm{i}}^{2} e^{2}}{\varepsilon_{0} m_{\mathrm{i}}} \approx \frac{n_{\mathrm{e}} Z_{\mathrm{eff}} e^{2}}{\varepsilon_{0} m_{\text {main }}},
\end{aligned}
$$

where $\omega$ is the wave frequency multiplied by $2 \pi, c$ is the light speed in vacuum and $v_{\mathrm{i}}=\sqrt{2 T_{\mathrm{i}} / m_{\mathrm{i}}}$ and $v_{\mathrm{e}}=\sqrt{2 T_{\mathrm{e}} / m_{\mathrm{e}}}$ are the most probable velocity of thermal ions and electrons (following notations of [9], equal to the thermal velocity multiplied by $\sqrt{2}$ ) of respective temperature $T_{\mathrm{i}}$ and $T_{\mathrm{e}} \cdot \omega_{\mathrm{pe}}$ is the electron plasma frequency and $\omega_{\mathrm{pi}}$ the one of the ions. In the expression of $\omega_{\mathrm{pi}}, e$ is the absolute value of the electron charge, $\varepsilon_{0}$ the dielectric permittivity of vacuum, $Z_{\text {eff }}$ the effective charge of the plasma, $m_{\mathrm{e}}$ the electron mass at rest and $m_{\text {main }}$ the mass of the main ion species.

The ray equations are solved in the spatial co-ordinates $(R, \phi, Z)$, respectively, major radius, toroidal angle, altitude with respect to the equatorial mid-plane, and their canonical 
variables $\left(k_{R}, n=R k_{\phi}, k_{Z}\right)$, where $\left(k_{R}, k_{\phi}, k_{Z}\right)$ are the components of the wave vector in the co-ordinate system $(R, \phi, Z)$ and $n$ is the toroidal mode number:

$$
\begin{array}{llll}
\frac{\mathrm{d} R}{\mathrm{~d} t}=-\frac{\partial D / \partial k_{R}}{\partial D / \partial \omega}, & \frac{\mathrm{d} \phi}{\mathrm{d} t}=-\frac{\partial D / \partial n_{\phi}}{\partial D / \partial \omega}, & \frac{\mathrm{d} Z}{\mathrm{~d} t}=-\frac{\partial D / \partial k_{Z}}{\partial D / \partial \omega} \\
\frac{\mathrm{d} k_{R}}{\mathrm{~d} t}=-\frac{\partial D / \partial R}{\partial D / \partial \omega}, & \frac{\mathrm{d} n_{\phi}}{\mathrm{d} t}=-\frac{\partial D / \partial \phi}{\partial D / \partial \omega}, & \frac{\mathrm{d} k_{Z}}{\mathrm{~d} t}=-\frac{\partial D / \partial Z}{\partial D / \partial \omega} .
\end{array}
$$

A fifth order Runge-Kutta algorithm with adaptative time step is used for calculating the ray trajectories [24]. The rays are computed for a given number of radial steps or are stopped before when the quasilinear electron Landau damping (ELD) criterion $n_{\|} \approx 6.5 / \sqrt{T_{\mathrm{e}}}$ is met with a significant margin, i.e. when $n_{\|} \geqslant 9 / \sqrt{T_{\mathrm{e}}}$.

The equilibrium magnetic field and the toroidal flux co-ordinate are mapped on a poloidal $(R, Z)$ grid, as input of the code, and numerically interpolated during the RT. This procedure allows us to use DELPHINE in any tokamak magnetic configuration and arbitrary shape of the current profile that is consistent with the magnetic equilibrium. In most cases, the magnetic equilibrium used in DELPHINE is directly given by a 2D solver, which provides calculations in a realistic geometry. The electron density and temperature profiles are mapped as a function of the toroidal flux co-ordinate and also interpolated numerically during the RT calculations. DELPHINE is able to take into account the toroidal magnetic field ripple in a perturbative way, using the method described in [15] and transposed in $(R, \phi, Z)$ geometry. The input $n_{\|}$ spectrum is usually limited to two Gaussian peaks but can be extended to any number of peaks, including negative $n_{\|}$contributions (for counter-current drive). It is decomposed into a series of rays, which are distributed both in $n_{\|}$and initial position, hence covering the $n_{\|}$spectrum and geometrical extent of the antenna, as prescribed in [11].

Once rays are calculated, the absorption module calculates the power damped resonantly on electrons along each ray according to the equation $[10,25]$

$$
\Delta P=-2 \gamma_{\mathrm{abs}} P \Delta t
$$

where $P$ is the power carried by the ray, $\Delta t$ is the propagation time interval between two calculated points of the ray. The absorption coefficient $\gamma_{\mathrm{abs}}$ is given by

$\gamma_{\mathrm{abs}}=\frac{\left[\left(\varepsilon_{\perp}-n_{\|}^{2}\right)\left(\varepsilon_{\perp}-n^{2}\right)-\varepsilon_{x y}^{2}\right]}{\partial D / \partial \omega}\left(-\left.\pi \operatorname{sgn}\left(k_{\|}\right) \frac{\omega_{\mathrm{pe}}^{2}}{k_{\|}^{2}} \gamma m_{\mathrm{e}}^{2} \frac{\partial f_{\|}}{\partial p_{\|}}\right|_{p_{\|}=\gamma m_{\mathrm{e}}\left(\omega / k_{\|}\right)}\right)$,

where $p_{\|}$is the electron momentum in the direction parallel to the local magnetic field, $\gamma$ is the relativistic factor evaluated at the resonance condition $v_{\|}=\omega / k_{\|}$and $f_{\|}$is the electron distribution function averaged over the perpendicular direction $f_{\|}=\int_{0}^{\infty} 2 \pi p_{\perp} \mathrm{d} p_{\perp} f$, with the electron distribution function $f$ normalized as $\int_{-\infty}^{\infty} \mathrm{d} p_{\|} \int_{0}^{\infty} 2 \pi p_{\perp} \mathrm{d} p_{\perp} f=1$. Note that expression (4) is derived assuming that most of the LH power is absorbed by fast electrons with $v_{\|} \gg v_{\perp}$, i.e. neglecting the dependence of $\gamma$ on $v_{\perp}$. This approximation allows us to use directly $f_{\|}$instead of integrating $\gamma f$ over the perpendicular direction for each evaluation of $\gamma_{\mathrm{abs}}$ and reduces significantly the computation time. Therefore $\gamma$ is evaluated in expression (4) using the relation $\gamma \approx 1 / \sqrt{1-\left(1 / n_{\|}^{2}\right)}$. For a typical Tore Supra case, this approximation leads to an underestimation of $\gamma_{\mathrm{abs}}$ of only $2 \%$, since the dominant contribution to the integral $\left.\int_{0}^{\infty} 2 \pi p_{\perp} \mathrm{d} p_{\perp} \gamma\left(\partial f / \partial p_{\|}\right)\right|_{p_{\|}=\gamma m_{\mathrm{e}}\left(\omega / k_{\|}\right)}$occurs indeed for small values of $v_{\perp}$ (typically $\left.v_{\perp} / v_{\mathrm{e}} \sim 1\right)$. Moreover, this already small underestimation of $\gamma_{\mathrm{abs}}$ is more or less compensated by the omission of the factor $\left[J_{0}\left(k_{\perp} p_{\perp} / m_{\mathrm{e}} \Omega_{c e}\right)\right]^{2}$ under the integral above, which is replaced 
by 1 in deriving expression (4) in the $p_{\perp} \approx 0$ approximation. See the appendix for the detail of the calculation of $\gamma_{\mathrm{abs}}$ and the quasilinear diffusion coefficient.

Following the usual method, the plasma is divided into several radial shells (typically 20). It is assumed that the electron distribution function depends only on the radius; therefore $f$ is constant within a radial shell. A local quasilinear diffusion coefficient is calculated for each shell. Each time step of each ray which is located in the given plasma shell contributes to the quasilinear diffusion coefficient, by adding to it:

$$
\begin{aligned}
\Delta D_{\mathrm{QL}}\left(p_{\|}, p_{\perp}\right) & =\frac{2 P \Delta t}{n_{\mathrm{e}} V} \frac{\left[\left(\varepsilon_{\perp}-n_{\|}^{2}\right)\left(\varepsilon_{\perp}-n^{2}\right)-\varepsilon_{x y}^{2}\right]}{\partial D / \partial \omega} \\
& \times\left[J_{0}\left(\frac{k_{\perp} p_{\perp}}{m_{\mathrm{e}} \Omega_{c e}}\right)\right]^{2}\left(\pi \frac{\omega_{\mathrm{pe}}^{2}}{\omega} \gamma m_{\mathrm{e}}^{2} \frac{1}{\left|k_{\|}\right|} \delta\left(p_{\|}-\gamma m_{\mathrm{e}} \frac{\omega}{k_{\|}}\right)\right),
\end{aligned}
$$

where $V$ is the volume of the plasma shell, $\gamma$ is the relativistic factor evaluated at the resonance condition as explained above. Expression (5) is derived so that the power corresponding to the quasilinear diffusion coefficient $\Delta D_{\mathrm{QL}}$ is equal to the power damped along one step of the ray, calculated from equations (3) and the full 2D expression of $\gamma_{\mathrm{abs}}$. The evaluation of the quasilinear diffusion coefficient therefore takes into account the $2 \mathrm{D}$ momentum dependence, with the relativistic factor $\gamma$ depending on $p_{\perp}$ and the factor $\left[J_{0}\left(k_{\perp} p_{\perp} / m_{\mathrm{e}} \Omega_{c e}\right)\right]^{2}$.

In order to evaluate expression (5) numerically, for a discrete 2D momentum grid, the Dirac distribution is replaced by a Gaussian with a fixed width $\Delta$ using the relation

$$
\delta(x)=\lim _{\Delta \rightarrow 0} \frac{1}{\Delta \sqrt{\pi}} \mathrm{e}^{-x^{2} / \Delta^{2}} .
$$

The spectral width of the resonance $\Delta$ is chosen equal to 0.1 . This value results as a trade-off between (i) providing a sufficiently smooth $D_{\mathrm{QL}}$ with respect to resolution of the momentum grid used in the FP code and (ii) avoiding spectral overlap between neighbouring rays. The latter constraint is critical not only for preventing the irrelevant acceleration of non-resonant electrons but also because it is a key element of the WKB assumptions. Indeed, rays can be considered independent only if the spectral overlap is negligible, since diffraction effects are then very small. This well-known limitation of the RT model can only be solved by considering a beam tracing approach when absorption is strong (single pass) [26, 27] or a full-wave description in weaker absorption regimes [28, 29]. It has been verified that the power deposition profile does not change significantly when varying $\Delta$ from 0.03 to 0.3 . This means that the code results are stable around the chosen $\Delta$ value, even if criterion (ii) becomes marginally satisfied as $\Delta$ increases.

The local FP equation which gives the electron distribution function in momentum space, neglecting any radial transport of fast electrons, may be expressed as

$$
\frac{\partial f}{\partial t}=\frac{\partial}{\partial p_{\|}} D_{\mathrm{QL}} \frac{\partial f}{\partial p_{\|}}+\left(\frac{\partial f}{\partial t}\right)_{\text {coll }}-E_{\|} \frac{\partial f}{\partial p_{\|}} .
$$

The first right hand term represents the quasilinear diffusion of electrons by the LH waves, the second term models the collisions with the various plasma species (electrons and ions) and the third term corresponds to the ohmic electric field. The FP module used in DELPHINE solves equation (7) in a 2D momentum space $(p, \xi)$, where $p$ is the modulus of the electron momentum and $\xi=p_{\|} / p$. It uses a symmetrization procedure in momentum space to account for the effect of particle trapping, using analytical bounce integrals for circular concentric magnetic 
flux surfaces [30]. The collision operator $(\partial f / \partial t)_{\text {coll }}$ is fully relativistic and corresponds to the results obtained in [31]. More details on the FP module can be found in [32]. In relations (4) and (5), the electron momentum distribution function used by the code corresponds to its value at the minimum of the magnetic field in the plasma equatorial mid-plane. Therefore, the poloidal dependence of $f$ is neglected as well as the bounce-averaging of the quasilinear diffusion operator. This simplified approach is again consistent with the assumption that electrons with large $p_{\perp}$ have a small contribution to the LH wave absorption process.

About eight iterations between the FP module and the absorption calculation are usually sufficient in order to converge towards consistent electron distribution function and LH power absorption in the various plasma shells. At each iteration, the FP solver evolves the distribution function on a rather long duration, of the order of $50 v^{-1}$ with respect to the reference time scale $v^{-1}$, where $v$ is the local electron collision frequency. The inverse time or fully implicit scheme used in the 2D FP solver allows us indeed to use integration time step much larger than $v^{-1}$. In principle, it is possible to take values larger than $50 v^{-1}$ in order to speed up the convergence and reduce the computer time consumption. If this turns out to be effective when power absorption takes place predominantly around one radial location, a too large time step may lead to bistable oscillations of the $\{\mathrm{FP}+$ absorption $\}$ calculation when power is spread into two main peaks for example. In that case, the power may be alternatively absorbed at one of the two peak positions. This is the consequence of the strong dependence of the power absorption process on the shape of the distribution in momentum space. Such a limitation can be only avoided by enforcing the consistency between $f$ and the quasilinear diffusion coefficient on a shorter time scale. The value $50 v^{-1}$ is then a trade-off between numerical stability and rate of convergence, and the wave damping can be correctly described at all radial positions without spurious behaviours, whatever the shape of the power deposition profile.

Once the iteration between the FP module and the absorption calculation has converged, the consistency of the result is checked by comparing the total LH power absorbed in each shellon the one hand $\left(P_{\mathrm{ABS}}\right)$ by summing the local contributions $\Delta P$ obtained from equation (3) and on the other hand $\left(P_{\mathrm{FP}}\right)$ by calculating the power corresponding to the quasilinear diffusion process:

$$
P_{\mathrm{FP}}=-2 \pi \int_{-1}^{+1} \mathrm{~d} \xi \int_{0}^{+\infty} \xi D_{\mathrm{QL}} \frac{\partial f}{\partial p_{\|}} \frac{p^{3}}{\gamma_{\mathrm{rel}}} \mathrm{d} p
$$

where $\gamma_{\text {rel }}$ is the relativistic factor corresponding to momentum $p$. Some deviation is unavoidable, mainly because it is numerically difficult to make a continuous description like a distribution function correspond to a discrete Dirac contribution (equation (6) is rigorously valid only for $\Delta \rightarrow 0$ ). When the convergence is successful, deviations $P_{\mathrm{ABS}}-P_{\mathrm{FP}}$ less than $20 \%$ of $P_{\mathrm{ABS}}$ are obtained and considered acceptable. However, owing to these deviations, the total absorbed power in the plasma as calculated by the FP module $\sum_{\text {shells }} P_{\mathrm{FP}} V$ can become slightly larger than the launched power $P_{\mathrm{LH}}$, while by construction $\sum_{\text {shells }} P_{\mathrm{ABS}} V=P_{\mathrm{LH}}$ in case of full absorption. In order to be consistent with the launched power when comparing with experiments, $P_{\mathrm{ABS}}$ is used as the power deposition profile, and the $\mathrm{LH}$ driven current $j_{\mathrm{LH}}$ is multiplied by the local value of $P_{\mathrm{ABS}} / P_{\mathrm{FP}}$, which conserves the local current drive efficiency calculated by the FP module. It is worth noting that the absorbed LH power $P_{\mathrm{FP}}$ is always consistent with the power transferred by collisions to the electrons $P_{\text {coll }}$ within the FP solver which indicates that this one runs consistently on its side without numerical problems. This condition is well fulfilled provided the value of $D_{\mathrm{QL}}$ never reaches too large values. Indeed, in that case, the matrix conditioning of the 2D FP solver becomes poor leading often to unphysical 


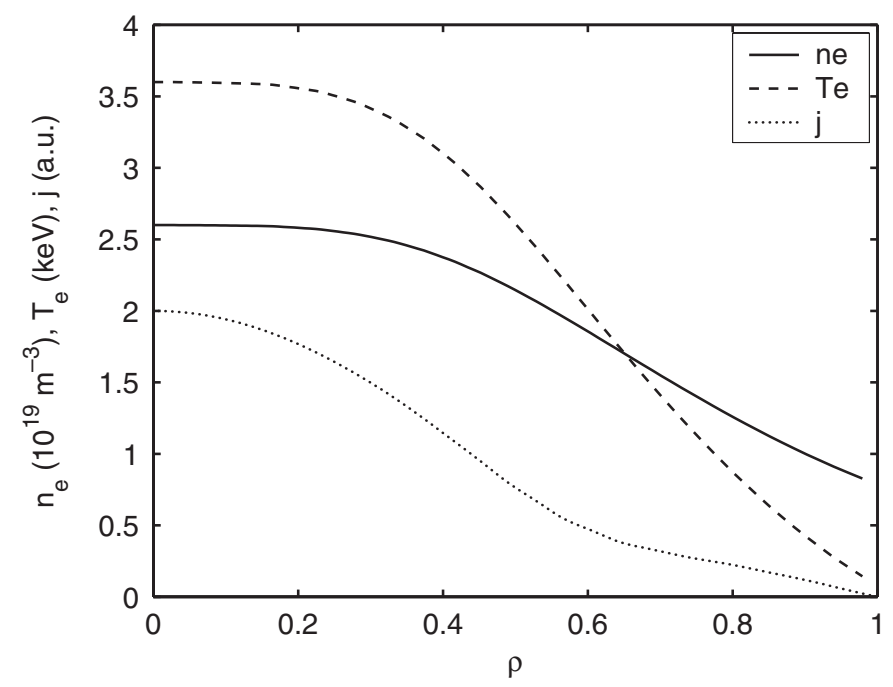

Figure 1. Profiles used for the computation of the equilibria: electron density $n_{\mathrm{e}}(-)$, electron temperature $T_{\mathrm{e}}(---)$, plasma current density $j(\cdots \cdots)$.

solutions with too large current drive efficiencies. Though $D_{\mathrm{QL}}$ may be in principle much larger than unity (in normalized units) from the physics point of view, its value is limited to 10 in this version of the code. This means that when $D_{\mathrm{QL}}\left(\rho, p_{\|}, p_{\perp}\right)$ reaches 10 , all following ray steps which would normally contribute to an additional $\Delta D_{\mathrm{QL}}$ at the same $\left(\rho, p_{\|}, p_{\perp}\right)$ have their $\gamma_{\text {abs }}$ set to zero. This approach is relevant since above $D_{\mathrm{QL}}=10$, the plateau formed in the distribution is already saturated, and the absorption of additional ray steps becomes negligible. A detailed discussion of the numerical problems for the LHCD may be found in [33].

The code outputs is the LH power deposition, the driven current and the electron distribution function (2D in momentum space, plus the radial dimension). The main calculation subroutines of the code are written in C (RT) or Fortran (FP solver), compiled into MEXfunctions to run in a Matlab ${ }^{\circledR}$ environment. All the other subroutines which do not require intensive and fast calculations are $\mathrm{Matlab}^{\circledR}$ functions, therefore the whole package is running under Matlab ${ }^{\circledR}$, taking advantage of its graphic and interactive features [34].

\section{Effect of plasma current on absorption and propagation of $\mathrm{LH}$ waves}

In order to investigate the dependence of the LH power deposition and driven current on the value of the total plasma current, a series of equilibria in the geometry of the Tore Supra tokamak have been prepared using the HELENA equilibrium solver [35]. Those equilibria have the same input pressure and normalized current density profiles (figure 1) but various values of the total current (0.4 to 1.4 MA). The propagation and absorption of the LH waves are computed using the DELPHINE code for each of these equilibria. The launched power spectrum is centred on $n_{\| 0}=2.0$ and discretized into 60 rays. This procedure allows us to determine the direct influence of the plasma current on the LH propagation and absorption, keeping all other parameters constant. It is usually not possible to do such a single-parameter scan in experiments, since for instance the temperature is expected to increase with the plasma current, owing to a better energy confinement. 

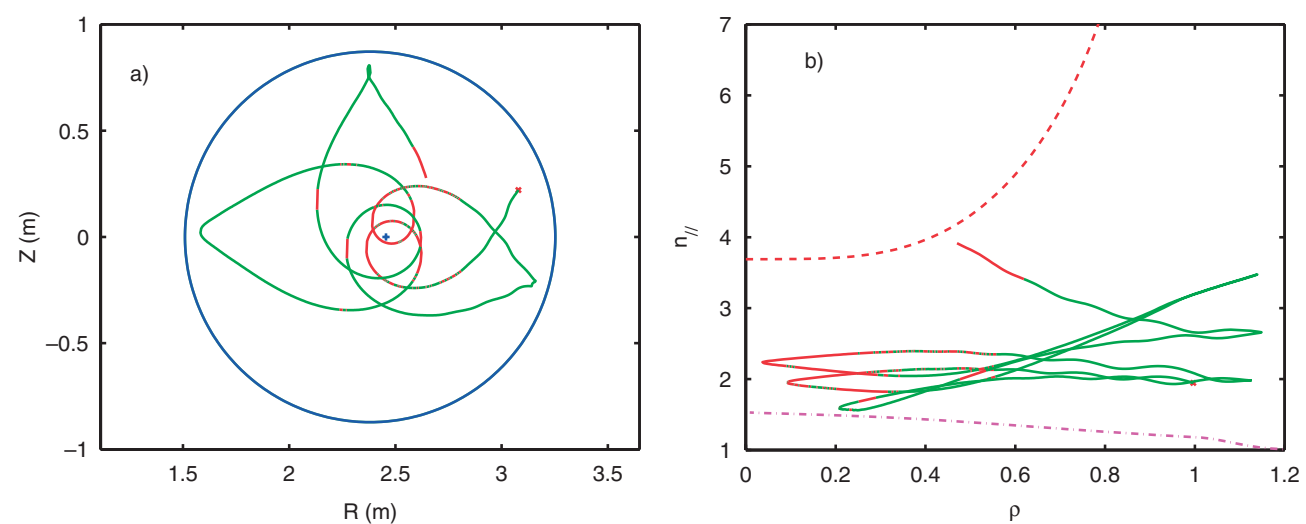

Figure 2. Propagation of a ray in the 1.4 MA configuration $(a)$ in the $(R, Z)$ co-ordinates, $(b)$ in the $\left(n_{\|}, \rho\right)$ co-ordinates. The red star shows the launching point of the ray and in $(a)$ the blue circle is the vacuum vessel and a blue '+' shows the magnetic axis. The ray is plotted with red dots where the absorption of the LH waves is significant, in green dots otherwise. In $(b)$ the red dashed line is the quasilinear Landau Damping criterion $n_{\|} \approx 6.5 / \sqrt{T_{\mathrm{e}}}$, the purple dash-dotted line is the accessibility limit $n_{\| \text {acc }}=\left(\omega_{\mathrm{pe}} / \Omega_{c e}\right)+\sqrt{\varepsilon_{\perp}}$.

In the configuration chosen here, which is typical of Tore Supra low density shots, the LH power deposition is strongly dependent on the detail of the wave propagation: first, the low density makes the whole plasma accessible to the LH waves. Second, the moderate values of the electron temperature $\left(T_{\mathrm{e} 0} \approx 3.6 \mathrm{keV}\right)$ and the fact that the $T_{\mathrm{e}}$ profile does not feature strong gradients make the absorption likely at any radial point inside $\rho=0.6$. Therefore, the localization of the power deposition is not known a priori and the results of the detail of the wave propagation as well. Moreover the simulations show that the rays do a few passes through the plasma before being absorbed. Hence neither the case of single-pass absorption (rays absorbed rapidly after their launch, which would occur for much larger electron temperature) nor that of multi-pass absorption (slow variation of $n_{\|}$per pass, so that the wave fills the entire propagation domain in the $\left(n_{\|}, \rho\right)$ space [36]) is achieved. In the former case, the propagation time is so short that the power deposition is determined almost completely by the wave characteristics at launch. In the latter, the absorption occurs necessarily at the intersection between the propagation domain and the Landau damping curve [36]. Conversely, in the few pass regime, the rays characteristics evolve rapidly and significantly between the launch and the absorption; therefore the propagation must be followed carefully in order to predict the location of the power deposition.

During the propagation, the parallel refractive index of some rays may increase owing to the toroidal geometry. These rays go through the spectral gap and drive a short electron tail just above the Maxwellian level. The condition for this linear ELD is approximately given by $n_{\|} \approx 6.5 / \sqrt{T_{\mathrm{e}}}$ [37]. Due to quasilinear effects, rays with lower $n_{\|}$are absorbed in turn by this suprathermal seed, which result in a fully developed suprathermal plateau in the electron distribution. Because the fast electron seed is very efficient at absorbing low $n_{\|}$rays, the quasilinear process leads often to narrow power deposition profiles, which peak at the position of the seed. As a consequence, the power deposition is mainly determined by the occurrence of high $n_{\|}$-upshift along some rays. A typical trajectory which provides high $n_{\|}$-upshift is when the ray propagates towards the edge density cut-off at the top of the chamber and then bounces back towards the plasma centre [8] (in fact, the sign of the $n_{\|}$variation depends on the direction of the rays rotation in the poloidal plane, e.g. if they turn counter-clockwise, $n_{\|}$-upshift occurs at the bottom of the chamber). Figure 2 shows the typical behaviour of a ray which starts 

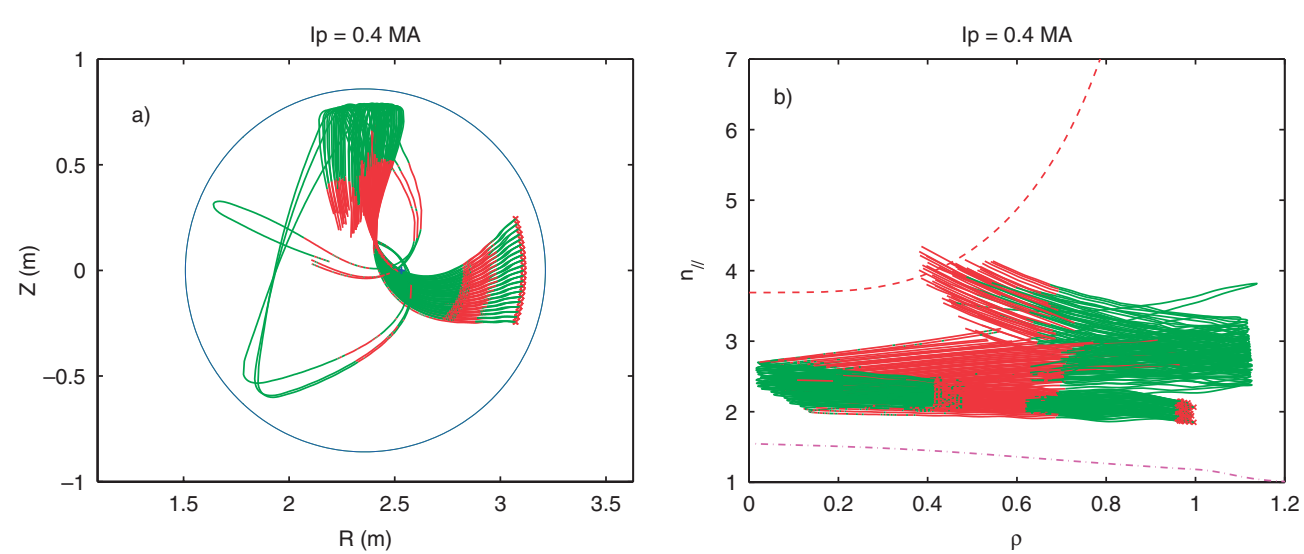

Figure 3. Propagation of the rays in the 0.4 MA configuration $(a)$ in the $(R, Z)$ co-ordinates, $(b)$ in the $\left(n_{\|}, \rho\right)$ co-ordinates. Same drawings and colour code as in figure 2.
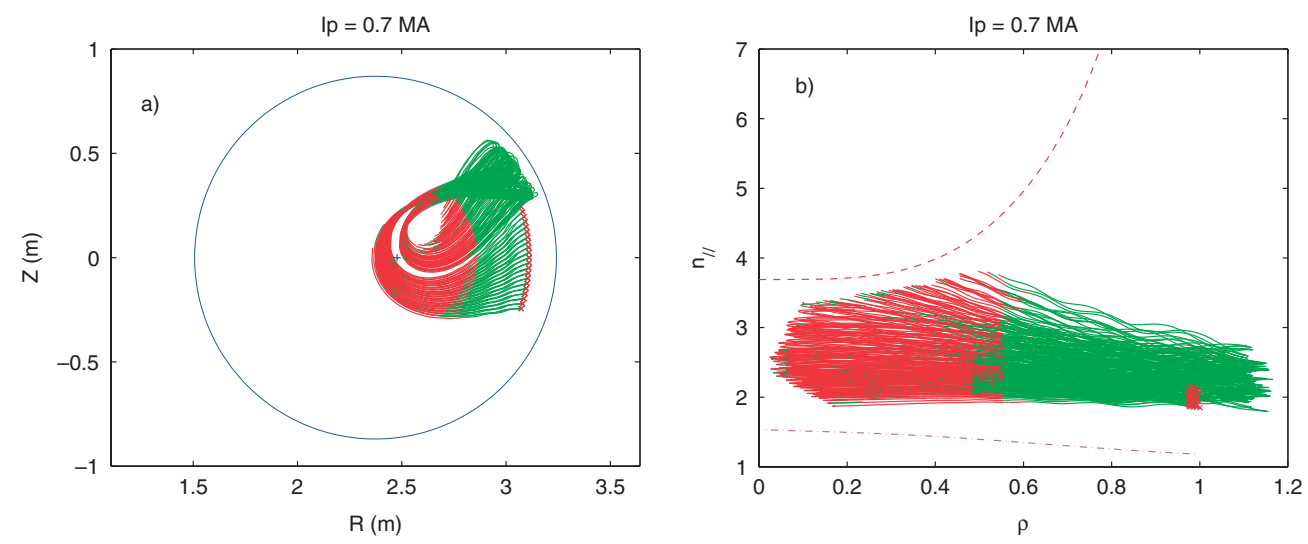

Figure 4. Propagation of the rays in the 0.7 MA configuration $(a)$ in the $(R, Z)$ co-ordinates, $(b)$ in the $\left(n_{\|}, \rho\right)$ co-ordinates. Same drawings and colour code as in figure 2.

doing a few passes near the equatorial mid-plane, with only a weak variation of its $n_{\|}$. Then, after it bounces back from the top of the vaccum vessel, its $n_{\|}$increases rapidly, and the ray is fully absorbed. Such rays tend to be damped off-axis, because their $n_{\|}$increases rapidly above the local ELD limit as they propagate towards the centre of the plasma (see figure 2). Conversely, rays with slower $n_{\|}$-upshift will first meet the ELD condition near the axis, where the electron temperature is usually maximum.

The propagation of the rays is clearly affected by the plasma current (see figures 3-5). The poloidal rotation of the rays increases as a function of the plasma current, as shown by the projections of the rays in the $(R, Z)$ space. Indeed, in the electrostatic approximation, the group velocity of the LH slow waves tends to be parallel to the local magnetic field [25]. Therefore the rays tend to follow the magnetic field lines, which have a higher rotational transform at higher plasma current. At $I_{\mathrm{p}}=0.4 \mathrm{MA}$ (figure 3), the rays, which are launched around the equatorial mid-plane from the low field side, do only a quarter of a turn in projection in a poloidal cross section before being absorbed. Since they travel through the top region of the plasma, their $n_{\|}$ undergoes a fast upshift. At $I_{\mathrm{p}}=0.7 \mathrm{MA}$ (figure 4), the rays do approximately half a turn in the poloidal cross section and stay around the equatorial mid-plane. As a consequence, their 

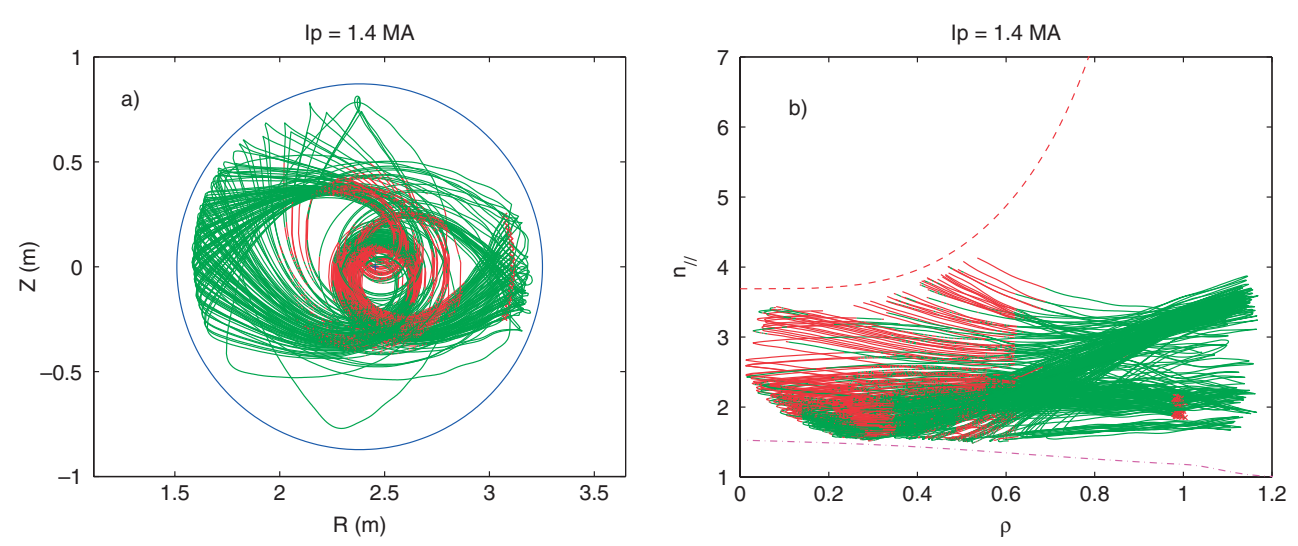

Figure 5. Propagation of the rays in the 1.4 MA configuration $(a)$ in the $(R, Z)$ co-ordinates, $(b)$ in the $\left(n_{\|}, \rho\right)$ co-ordinates. Same drawings and colour code as in figure 2.

$n_{\|}$-upshift is slower. At $I_{\mathrm{p}}=1.4 \mathrm{MA}$ (figure 5), the rays rotate faster and are being spread also much more through the poloidal cross section. From one ray to the other, quite different kinds of propagation are obtained: some rays undergo slow $n_{\|}$-upshift and are absorbed preferentially near the magnetic axis, while the others undergoing fast $n_{\|}$-upshift are absorbed in a more outer region $(\rho=0.4-0.6)$. This behaviour is thought to be at the origin of the broadening of the measured hard-x rays profiles at high plasma current, as reported in [18]. In an experiment, this effect is amplified by the simultaneous increase of electron temperature and energy confinement with the plasma current. Indeed, increasing and broadening the electron temperature profile will move the power deposition of fast $n_{\|}$-upshifted rays more off-axis. Conversely, for colder plasmas (on-axis $T_{\mathrm{e}} \leqslant 2 \mathrm{keV}$ ) with narrow $T_{\mathrm{e}}$ profile, the ELD limit is so high off-axis that even such rays can reach it only close to the axis. This explains why the measured FEB emission is usually narrow at $I_{\mathrm{p}}=0.4 \mathrm{MA}$ on Tore Supra, in spite of the fast $n_{\|}$-upshift predicted in the present simulation.

The plasma current is also found to influence the lower bound of the $n_{\|}$of the rays bundle, which is a determinant factor for the current drive efficiency since it corresponds to the maximum velocity of the accelerated electrons. At low $I_{\mathrm{p}}$ (figure 3 ), the $n_{\|}$of the rays increases during their first pass from the launching point to the plasma centre, while it decreases at high $I_{\mathrm{p}}$ (figure 5). As a consequence, the minimum $n_{\|}$reached by the rays is found to decrease with increasing plasma current. Then, the rays undergo $n_{\|}$-upshift, which can be quite strong in the high plasma current equilibrium $\left(I_{\mathrm{p}}=1.4 \mathrm{MA}\right.$, see figure 5 and previous paragraph), hence providing an efficient filling of the spectral gap, even off-axis. These conditions are ideal to form a continuous quasilinear plateau in the electron distribution function, whose high velocity limit increases with the plasma current, resulting in a higher current drive efficiency (figure 6).

Note that such a dependence can be qualitatively expected when considering the lower bound of the LH waves propagation domain [38]. In the electrostatic limit and doing a development to the first order in $B_{\theta} / B$ (where $B_{\theta}$ is the poloidal magnetic field and $B$ the total magnetic field), the lower $k_{\|}$boundary of the wave propagation domain is given by

$$
k_{\| \min } \approx \frac{n / R}{1+\left(B_{\theta} / B\right)\left(\omega_{\mathrm{pe}} / \omega\right)} .
$$

From this equation, the lower boundary decreases when the plasma current increases, through $B_{\theta}$ in the denominator. The present RT simulations show a similar dependence but in a stronger 


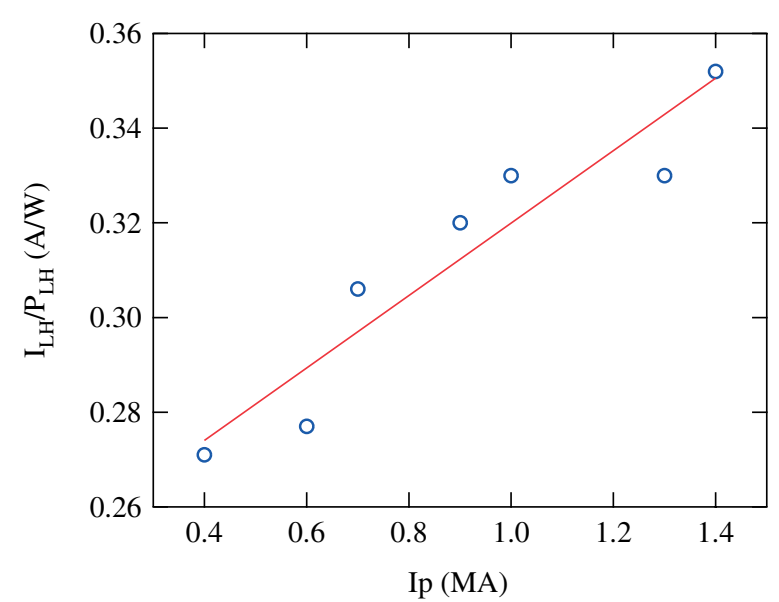

Figure 6. Global current drive efficiency (total LH driven current divided by the injected LH power) as a function of the plasma current, obtained from the simulations (circles). These cases feature full absorption of the LH power, i.e. all the injected power is absorbed by electron Landau damping. The solid red line shows the linear least-square fit to the simulation results.

way. Indeed, the rays do not reach necessarily the lower bound of the propagation domain. This is particularly true for the $I_{\mathrm{p}}=0.4 \mathrm{MA}$ equilibrium, where the rays $n_{\|}$increase almost constantly until full absorption. Therefore the dependence on plasma current of the minimum $n_{\|}$of the rays bundle predicted by the RT simulations is stronger than the variation of the lower bound of the propagation domain. This stresses the importance of the RT approach in cases where the waves do only a few passes before being absorbed and do not fill the whole propagation domain.

As shown in figure 6, an offset linear dependence of the current drive efficiency on the plasma current is obtained from the simulations. This effect appears more as a global trend than an accurate and systematic rule. Indeed the behaviour of the rays is quite complex, and the resulting detailed dependences cannot be captured in a simple picture. The power deposition profile changes with plasma current, which can affect the current drive efficiency since the electron temperature and density are not constant through the plasma. However the main trend deduced from figure 6 (offset linear dependence of $\eta_{\mathrm{LH}}$ on $I_{\mathrm{p}}$ ) is ascribed to the spectral characteristics of the rays at the location of absorption. Indeed, the local current drive efficiency increases with the plasma current, as shown in figure 7. Therefore the main behaviour of the global efficiency is not due to variations of the radial power deposition profile but to the local LH wave spectrum. For instance, both the $I_{\mathrm{p}}=0.4 \mathrm{MA}$ and $I_{\mathrm{p}}=0.7 \mathrm{MA}$ cases feature a minimum $n_{\|}$of about 1.9 in the absorbed wave spectrum, but the $I_{\mathrm{p}}=0.4 \mathrm{MA}$ case has a lower current drive efficiency because a gap remains around $n_{\|}=3$ in the ray distribution (see figure $3(b)$, around $\rho=0.5$ ). This gap induces a break in the quasilinear plateau of the electron distribution, i.e. the density of suprathermal electrons drops for velocities above $c / 3$. The high current case $I_{\mathrm{p}}=1.4 \mathrm{MA}$ features both a low value of the minimum $n_{\|}(\sim 1.5)$ in the absorbed wave spectrum and a continuous filling of the spectral gap, as explained above. Therefore it features a high local current drive efficiency, 10 to $20 \%$ higher than the $I_{\mathrm{p}}=0.7$ MA case in the region $0.1<\rho<0.6$ (figure 7).

The dependence of $\eta_{\mathrm{LH}}$ on $I_{\mathrm{p}}$ deduced from the simulations is qualitatively consistent with the experimental observations reported in figure $6(b)$ of [18]. Moreover, the interpretation in terms of increase of the upper velocity limit of the quasilinear plateau with increasing plasma current is fully consistent with the experimental observations. The photon temperature of 


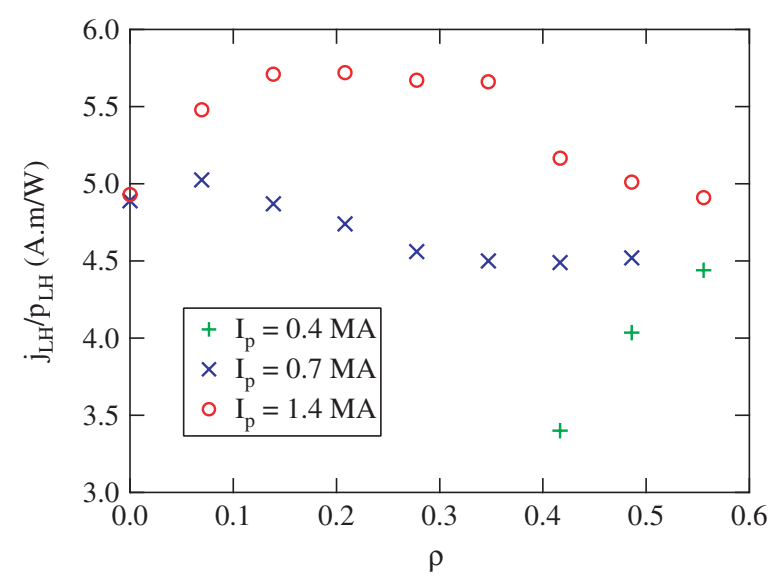

Figure 7. Ratio of the local $\mathrm{LH}$ driven current density (in $\mathrm{A} / \mathrm{m}^{2}$ ) to the local power deposition (in $\mathrm{W} / \mathrm{m}^{3}$ ), as a function of the normalized toroidal flux co-ordinate, obtained from the simulations at $I_{\mathrm{p}}=0.4(+), 0.7(\times)$ and $1.4 \mathrm{MA}(\mathrm{O})$. This local current drive efficiency is calculated where the main part of the power is deposited (i.e. where the power deposition is above $10 \%$ of its peak value).

the FEB and the energy of the fast electron ripple losses have been found to increase with $I_{\mathrm{p}}$, which is experimental evidence of the extension of the quasilinear plateau towards higher velocities [39]. The simulations show that the $I_{\mathrm{p}}$ dependence of the wave propagation can explain this behaviour, at least qualitatively.

Quantitatively, the magnitude of the effect is less important in the simulations than in the experimental data: between $I_{\mathrm{p}}=0.4$ and $I_{\mathrm{p}}=0.8 \mathrm{MA}$, the current drive efficiency increase is of the order of $12 \%$ from the simulation, while it is of the order of $33 \%$ in the experimental case. Despite the large error bars on this experimental value, mainly due to uncertainty on the effective charge $Z_{\text {eff }}$, this suggests that other plasma parameters could amplify the trend due only to the plasma current in the simulations. For instance, the electron temperature increases with $I_{\mathrm{p}}$, and it is expected that the LHCD efficiency also increases with the electron temperature $[40,41]$. In the simulations, the electron temperature has been kept constant in order to isolate the effect of the plasma current on the power deposition profile. Another source of inconsistency between the simulations and the experiments is that a prescribed normalized plasma current density profile has been used. The experiments which showed the current drive efficiency dependence on $I_{\mathrm{p}}$ have been carried out at zero loop voltage. This means that the plasma current density profile should be almost identical to the LH driven current density, the typical bootstrap fraction being of the order of $10-15 \%$. This may be a source of discrepancy in the magnitude of the effect between the simulations and the experiments. In the next two sections of this paper, some issues related to the calculation of the self-consistent solution for zero loop voltage discharges with dominant LHCD are addressed.

In summary, these RT/FP simulations capture, at least partially, the trend of increasing current drive efficiency with increasing plasma current, which has been observed on Tore Supra using a dataset of zero loop voltage steady-state discharges. The phenomenon is explained by the decrease of the minimum $n_{\|}$reached by the quasilinear plateau as a result of the rays propagation, which is more important than what could be deduced by considering only the wave propagation domain. This interpretation implies an upper limit for this dependence: in any case, the $n_{\|}$cannot go below the accessibility limit of the waves, which depends essentially on the electron density and the toroidal field $\left(n_{\| \text {acc }}=\left(\omega_{\mathrm{pe}} / \Omega_{c e}\right)+\sqrt{\varepsilon_{\perp}}\right.$, see, e.g. [25]). 

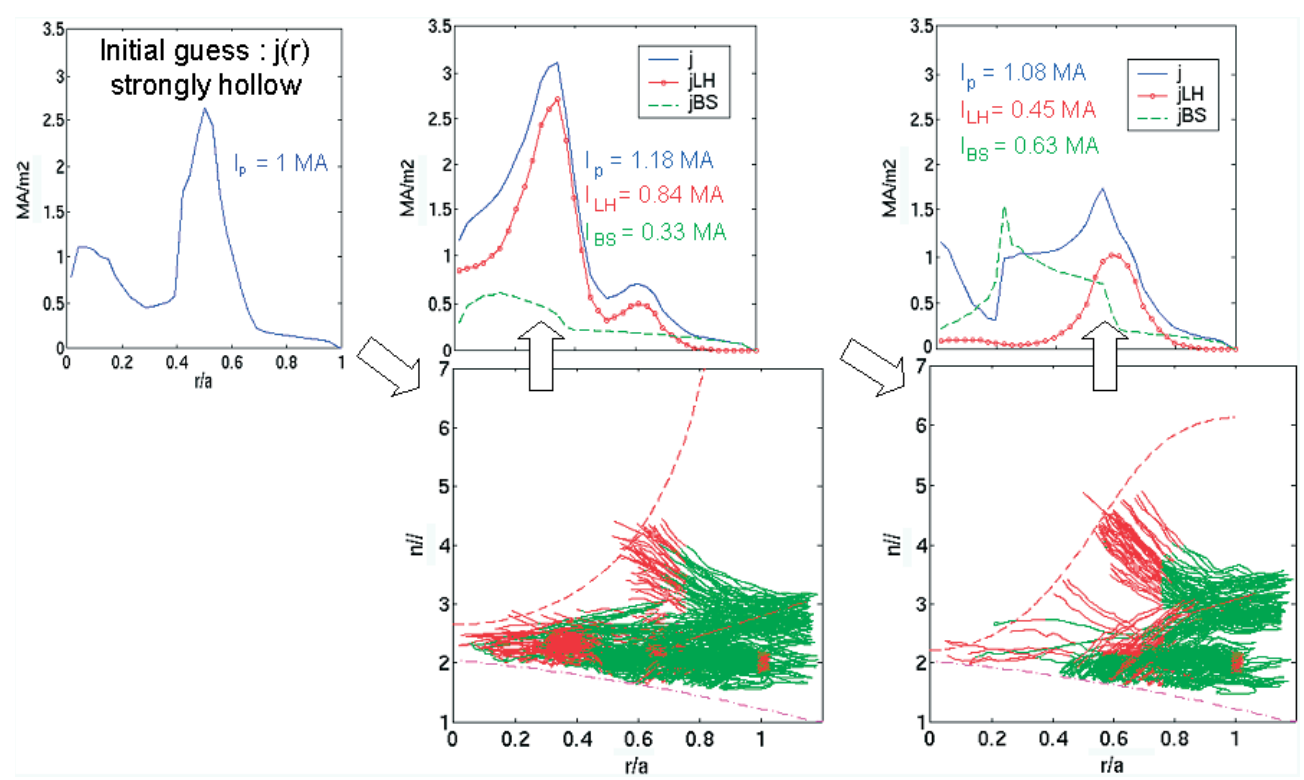

Figure 8. Successive steps (from left to right) of the DELPHINE/ASTRA convergence procedure, using $n_{\| 0}=2.0$. Bottom row: propagation of the rays in the $\left(n_{\|}, \rho\right)$ co-ordinates, calculated by DELPHINE using the equilibrium current density of the previous column. Top row: current density profiles calculated by ASTRA using the LH power deposition given by DELPHINE (graph from below in the same column), - - - -: bootstrap, $\bigcirc: \mathrm{LH}, \ldots$; total.

Therefore the decrease of the minimum $n_{\|}$with increasing plasma current will stop at the accessibility limit, and no further increase of current drive efficiency can be expected. Some other mechanisms which may also limit this effect are discussed in [39].

\section{Effect of $q$-profile shape on the propagation and absorption of LH waves}

In the previous paragraph, we have analysed the influence of the current profile on the LH waves propagation, varying the total plasma current while keeping the shape of the profile constant. We address now the issue of varying the current density profile at constant plasma current.

Figure 8 shows DELPHINE simulations related to high power and high density deuterium discharges, whose parameters correspond to the forecoming upgrade of Tore Supra heating systems (CIMES project [42]): volume-averaged electron density $\left\langle n_{\mathrm{e}}\right\rangle=5.0 \times 10^{+19} \mathrm{~m}^{-3}$, effective charge $Z_{\text {eff }}=1.72$, toroidal field $B_{\mathrm{t}}=4 \mathrm{~T}$, major radius $R=2.4 \mathrm{~m}$, minor radius $a=0.72 \mathrm{~m}$ and circular cross section. Heating and current drive powers are $\mathrm{P}_{\mathrm{ICRH}}=10 \mathrm{MW}$ (hydrogen minority heating scheme, $50 \%$ of power deposited on electrons, broad central deposition), $\mathrm{P}_{\mathrm{ECRH}}=2 \mathrm{MW}$ (central deposition) and $\mathrm{P}_{\mathrm{LH}}=12 \mathrm{MW}$ (3 grills) with $60 \%$ of the LH power contained in the main peak of the launched $n_{\|}$-spectrum, whose central value $n_{\| 0}$ is equal to 2.0. These discharges are expected to be carried out at zero loop voltage, purely driven by LHCD and bootstrap current. In order to obtain an equilibrium and energy confinement consistent with the non-inductively driven current, the ASTRA transport code [43] is used to recalculate them, given the LH power deposition calculated by DELPHINE as input. Since DELPHINE is not directly coupled to ASTRA, the following coupling method is used: ASTRA calculates a standard plasma evolution towards a steady-state with fixed LH power deposition 
and driven current density profiles, as given by the RT/FP code. The obtained steady-state plasma equilibrium and electron temperature profile are then used as input in DELPHINE to calculate a new LH current and power deposition. Note that this coupling method is rather crude, especially for LHCD dominated discharges and is presented here only to illustrate the impact of magnetic shear on the LH wave propagation and absorption. Accurate integrated modelling strategies for LHCD dominated discharges are discussed below in section 5. Though radial diffusion of fast electrons is likely to be negligible in such plasmas [44], these LH profiles are smoothed using an ad hoc radial diffusion coefficient of the order of $0.5 \mathrm{~m}^{2} \mathrm{~s}^{-1}$. This is not only done mainly to avoid the formation of too strong internal transport barriers in combination with the transport model described below, but also to account for the fact that measured LH power deposition profiles are systematically broader than the prediction of standard RT/FP codes $[18,45]$. The smoothed LH profiles are then given as input for a new ASTRA calculation. This process is iterated until a constant LH power deposition profile is reached. In ASTRA calculations, the density profile is kept constant, and electron heat transport is computed using the mixed Bohm-gyroBohm model with shear function described in [6]. This model takes into account a reduction of the electron heat diffusion coefficient for low or negative magnetic shear and has been extensively validated on Tore Supra. Bootstrap current is self-consistently calculated in ASTRA using the Hirschmann model [46] and represents 30 to $60 \%$ of the total current in these simulations, the rest of the current being driven by LH waves. The total plasma current remains nearly constant during the successive steps of the convergence procedure, at a value of 1.1 MA (relative variation lower than $10 \%$ ).

Comparing the successive steps of this convergence procedure provides clear evidence of the influence of the $q$-profile shape of the LH waves propagation and absorption, at fixed plasma current. Starting from a rather hollow current density profile (maximum at normalized radius $\rho=0.5$ ), the calculated LH power deposition is more central (max. at $\rho=0.35$ ). In turn, starting from this more peaked current density profile, the updated LH power deposition becomes strongly hollow (max. at $\rho=0.6$ ). The influence of the current density profile is clearly dominant with respect to the electron temperature, as shown in the $\left(n_{\|}, \rho\right)$ propagation diagrams, where the characteristics of the $n_{\|}$-upshift of the rays are modified by the change of the current profile while the ELD curve stays approximately at the same position. As shown in figure 8 , the $n_{\|}$-upshift is rather fast (resp. slow) when the plasma equilibrium has a peaked (resp. hollow) current density profile, which provides off-axis (resp. central) absorption. The mechanism at work here is similar to the one described in section 3, i.e. the influence of the local poloidal magnetic field on the ray trajectories in the poloidal cross section and its consequences on the toroidal $n_{\|}$-upshift. Note that this dependence of the rate of the $n_{\|}$ variation on the magnetic shear can be predicted in the case of the electrostatic dispersion relation using the numerical mapping derived in [16].

Hence LHCD systematically tends to broaden and smooth the initial current density profile, whatever its shape. In discharges dominated by LHCD, this gives rise to a self-regulating mechanism of the LH power deposition, through its dependence on the total current density profile. From the point of view of tokamak operation, a major consequence of this process is the reduction of the current profile control capability expected by tuning the launched $n_{\|}$-spectrum. One could also expect such a non-linear mechanism to play a role in the periodic electron temperature oscillation regime recently discovered in Tore Supra during steady-state LHCD discharges ('O-regime' $[47,48]$ ). Of course, in an experiment, the coupled LH and total current density profiles evolve in a continuous way and do not feature the large jumps observed between successive steps of the crude and indirect coupling method used in this section. Nevertheless, these results emphasize the need for a more accurate integrated modelling strategy for LHCD dominated discharges, which is discussed below. 


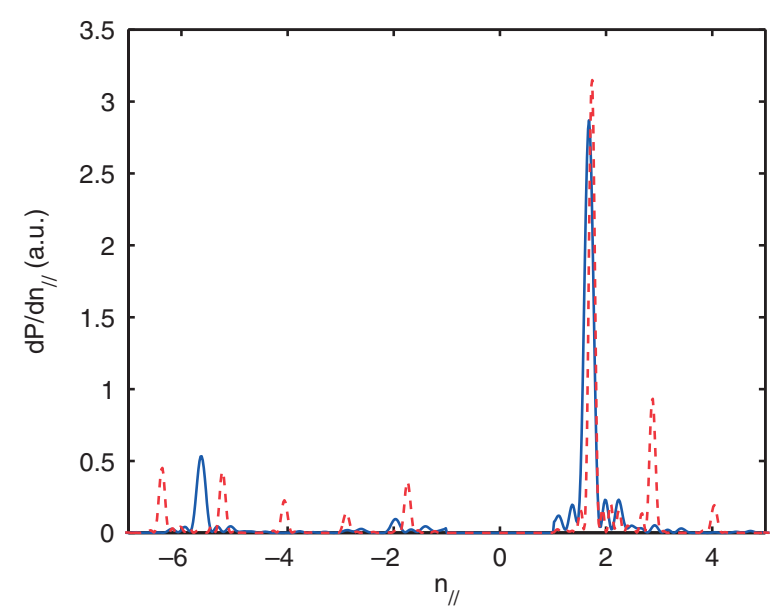

Figure 9. Power spectra of the two LH launchers of Tore Supra for shot \#32299, as calculated by the SWAN code.

\section{Implications for self-consistent modelling of discharges dominantly driven by LH waves}

In discharges where most of the current is driven by LH waves, the total current profile is close to the LHCD profile. Since, as shown in the previous paragraph, the LHCD profile itself depends on the total current profile, the self-consistency of LHCD and the total current density profile is essential for the modelling of such discharges. Several integrated modelling codes (e.g. ASTRA, JETTO) now include a direct coupling of the transport equations to a RT/FP solver (see for instance [23,49]). In a similar way, the RT/FP package DELPHINE has been coupled to the CRONOS integrated modelling code [50], which allows us to do simulations where the transport properties, the plasma equilibrium and the various sources and sinks (heating, current drive, particles, rotation ...) are calculated in a fully consistent way. Therefore, it is possible to carry out LHCD calculations with DELPHINE in a time dependent transport simulation, during which the current profile is evolving due to the resistive diffusion and possible variations of the current sources. In this paragraph, we show that the high sensitivity of LHCD on the total current profile puts strong constraints on the rate at which the LHCD package must be called, in order to converge towards the self-consistent solution.

Our analysis is based on a simulation of Tore Supra discharge \#32299, the longest pulse achieved up to now in this tokamak, with a world record in injected then exhausted energy of the plasma of more than 1 GJ [51]. The discharge remains in pure steady state for more than 6 min, with zero loop voltage enforced by feedback loop on the transformer. The plasma current is sustained at $80 \%$ by means of $2.9 \mathrm{MW} \mathrm{LHCD}$ (with peak refractive parallel index $n_{\| 0}=1.7$ ), the remaining $20 \%$ being driven by bootsrap current. The plasma has the following characteristics: main gas deuterium, circular cross section, major radius $R_{0}=2.37 \mathrm{~m}$, minor radius $a=0.70 \mathrm{~m}$, central electron density $n_{\mathrm{e} 0}=2.6 \times 10^{+19} \mathrm{~m}^{-3}$, on-axis toroidal magnetic field $B_{\mathrm{t}}=3.4 \mathrm{~T}$ and plasma current $I_{\mathrm{p}}=0.5 \mathrm{MA}$. The full launched $n_{\|}$spectrum of the LH waves is used in the RT calculation, as estimated by the SWAN code [52] (figure 9). The power deposition profiles from DELPHINE are slightly smoothed with a radial diffusion coefficient of fast electrons of $0.1 \mathrm{~m}^{2} \mathrm{~s}^{-1}$, in order to get rid of sharp peaks which may cause numerical 


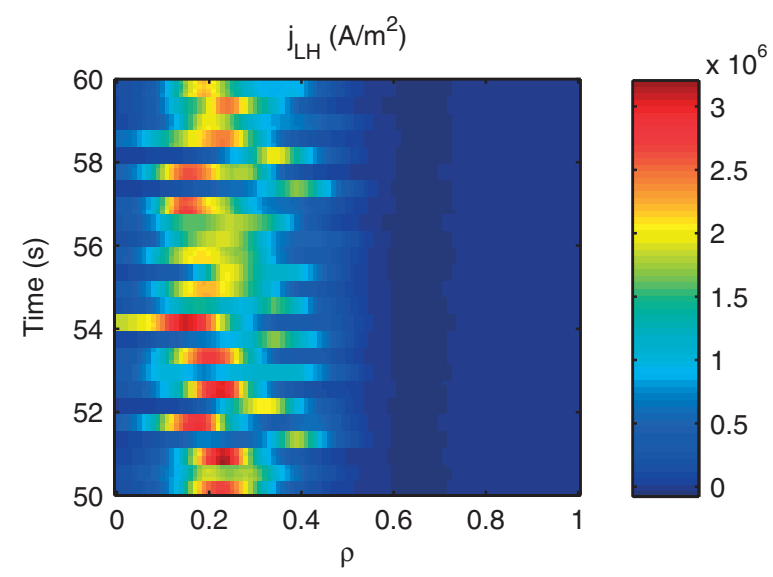

Figure 10. Time evolution of the LH driven current density profile, CRONOS simulation of Tore Supra shot \#32299, using 100 rays per antenna and call to DELPHINE every $0.4 \mathrm{~s}$.

difficulties in the current diffusion. Notice that such a small diffusion coefficient does not broaden significantly the LH power deposition. Finally, the LH driven current density profile is normalized in order to impose a constant total LH current, corresponding to $80 \%$ of the plasma current.

In a first simulation, DELPHINE is called every $0.4 \mathrm{~s}$. The RT/FP calculation uses 100 rays for each antenna (there are $2 \mathrm{LH}$ launchers in Tore Supra), which represents a step between two rays of $\Delta n_{\|}=2 \times 10^{-2}$. The current diffusion is calculated during $10 \mathrm{~s}$, all the other profiles (density, temperatures) being taken from the experiment and stationary. As shown in figure 10, the LH power deposition is not fully stabilized, even after $10 \mathrm{~s}$. The position of its maximum oscillates between $\rho=0.15$ and 0.4 , reflecting in this time-dependent simulation the trend described in the previous paragraph with stationary solutions: a slightly more peaked $\mathrm{j}_{\mathrm{LH}}$ profile yields a more peaked total current profile, which in turn provides a more hollow $\mathrm{j}_{\mathrm{LH}}$ profile at the next RT/FP calculation (figure 11). As a consequence, the internal inductance also oscillates between 1.9 and 2.0. In order to stabilize this bi-stable behaviour, we now increase the calling rate to DELPHINE, as well as the number of rays used in the calculation.

In this second simulation, DELPHINE is called every $0.1 \mathrm{~s}$ and uses about 400 rays for each antenna, which represents a spacing between two rays of $\Delta n_{\|}=5 \times 10^{-3}$ (figure 12). As shown in figure 13, the internal inductance is now stabilized after $4 \mathrm{~s}$ of current diffusion, at a value of 2.05. The amplitude of variation of the position of the peak deposition is much smaller than in the previous case, i.e. it varies between $\rho=0.1$ and $\rho=0.25$. In those conditions, the maximum variation of safety factor $q$ at $\rho=0.2$ (in the vicinity of LH maximum deposition) after $t=54 \mathrm{~s}$ is only of 0.1 , while it is of the order of 0.6 in the previous case (figure 14). Therefore a quasi-stationary state is obtained, within this quite satisfying error bar on the $q$-profile.

It becomes possible then to compare this result with the prediction of $q$-profile obtained using the bremsstrahlung emission of fast electrons as a measurement of the LH power deposition profile and driven current. This simple assumption is justified by the fact that when reconstructing the FEB emission from an FP calculated electron distribution function, the three profiles have very similar shapes. Therefore a reference CRONOS simulation is done using as $j_{\mathrm{LH}}$ the emission profile measured by FEB tomography [21], normalized to the 

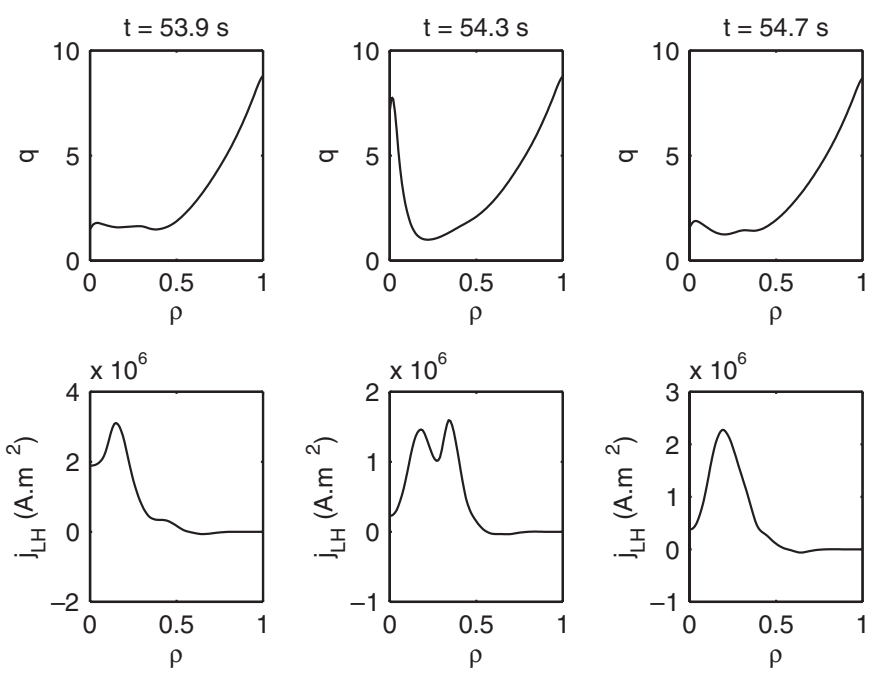

Figure 11. CRONOS simulation of Tore Supra shot \#32299, using 100 rays per antenna and call to DELPHINE every $0.4 \mathrm{~s}$. Top row: $q$-profile for $t=53.9,54.3$ and $54.7 \mathrm{~s}$, resulting from the current diffusion. Bottom row: LH-driven current density calculated by DELPHINE using the $q$-profile just above.

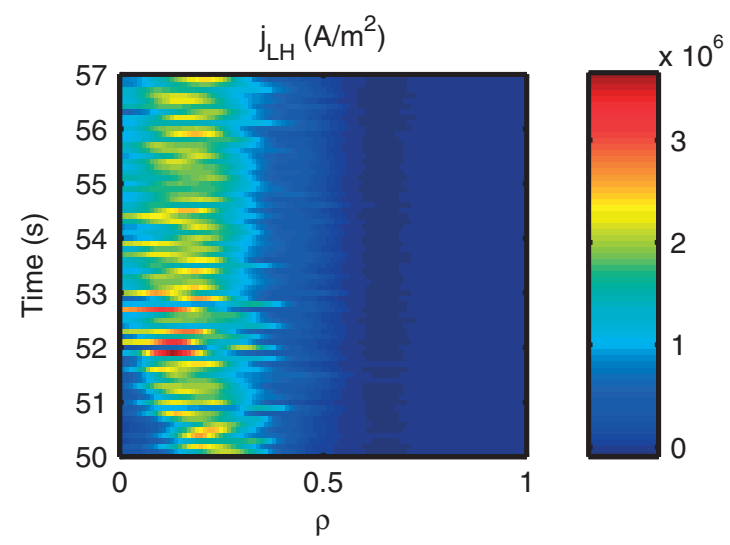

Figure 12. Time evolution of the LH driven current density profile, CRONOS simulation of Tore Supra shot \#32299, using 400 rays per antenna and call to DELPHINE every $0.1 \mathrm{~s}$.

relevant constant current drive efficiency in order to obtain zero loop voltage. The method is also a fortiori validated since it yields an internal inductance in excellent agreement with the experimental one ( $1.69 \pm 0.02$ in the simulation against $1.70 \pm 0.05$ measured $)$ and a $q$-profile compatible with a $3 / 2$ MHD mode which is observed experimentally throughout the discharge (figure 15). As shown in figure 16, the $j_{\text {LH }}$ profile deduced from FEB and the one calculated by DELPHINE have similar shapes: both have their maximum at the same position $\rho=0.2$ and comparable slope in their gradient region $(\rho=0.3-0.4)$. The DELPHINE $j_{\mathrm{LH}}$ exhibits a negative bump around $\rho=0.65$, due to the negative high $\left|n_{\|}\right|$component of the $n_{\|}$ spectrum. This bump is positive on the FEB-deduced $j_{\mathrm{LH}}$, since by definition we assume only positive current drive. This difference around $\rho=0.65$ has a significant impact on the value of $j_{\mathrm{LH}}$ around $\rho=0.2$, since the profiles are normalized to produce the same total current 


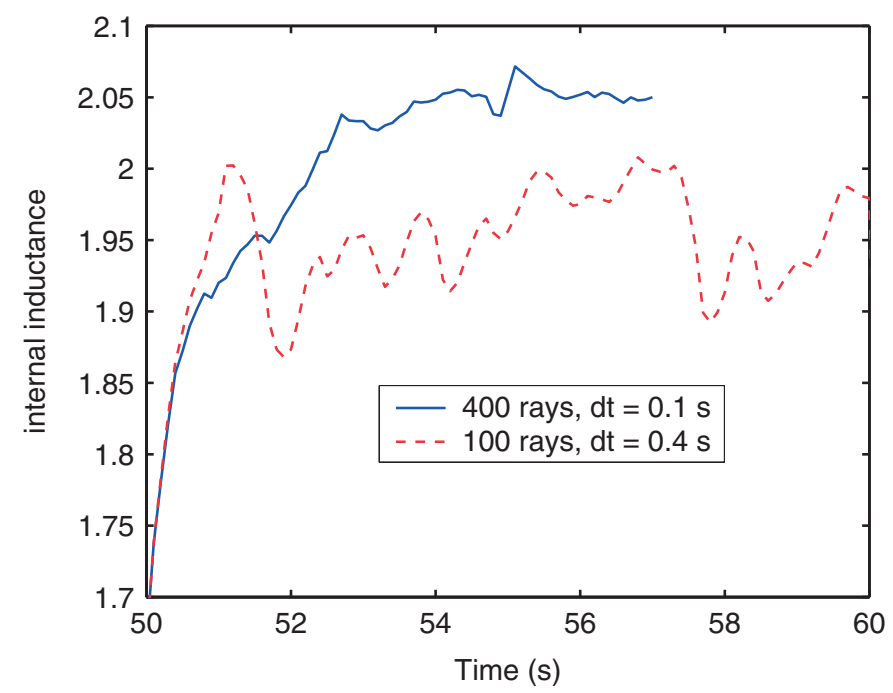

Figure 13. Time evolution of the internal inductance, CRONOS simulation of Tore Supra shot \#32299, for the case with 400 rays per antenna, call to DELPHINE every $0.1 \mathrm{~s}$ (solid, blue), and 100 rays per antenna, call to DELPHINE every $0.4 \mathrm{~s}$ (dash, red).

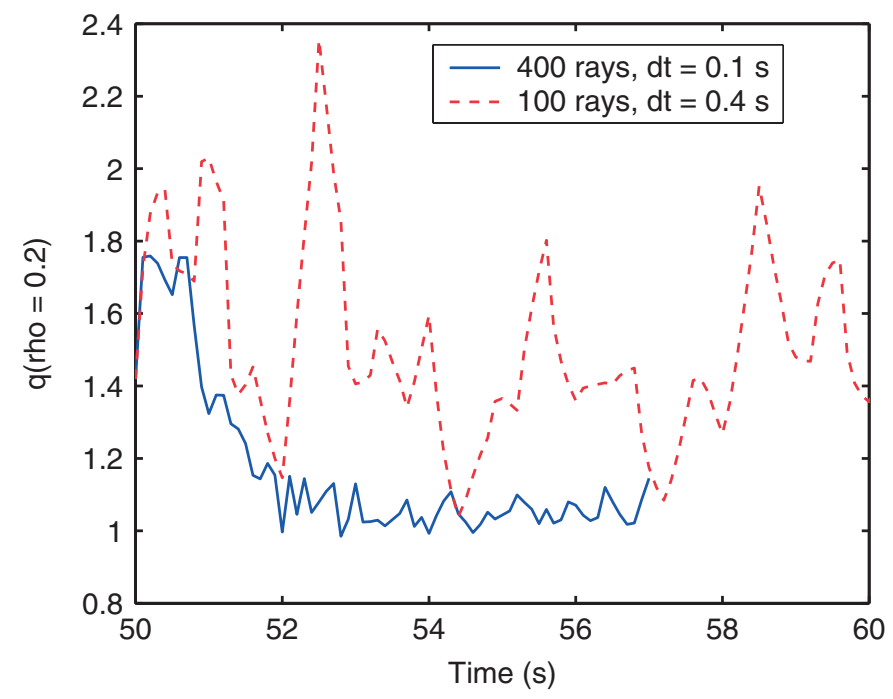

Figure 14. Time evolution of $q$ at $\rho=0.2$, CRONOS simulation of Tore Supra shot \#32299, for the case with 400 rays per antenna, call to DELPHINE every $0.1 \mathrm{~s}$ (solid, blue), and 100 rays per antenna, call to DELPHINE every $0.4 \mathrm{~s}$ (dash, red).

( $j_{\mathrm{LH}}$ integrated over the whole poloidal cross section). In spite of this effect on the absolute values of $j_{\mathrm{LH}}$, the shapes of the profiles are quite similar around their maximum. The excellent agreement of the FEB-deduced current profile with the measured internal inductance and MHD behaviour of this shot suggests that this one is closer to reality than the DELPHINE calculation. The main conclusion from this comparison is that it is needed to use the full $n_{\|}$spectrum in order to obtain the correct width of the power deposition (no power deposition at $\rho=0.6$ is 


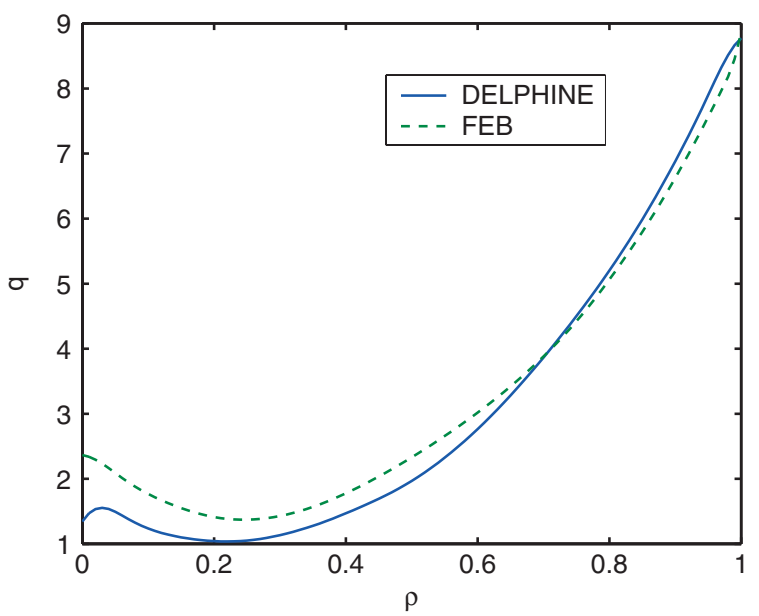

Figure 15. $q$-profile in the steady-state phase of shot Tore Supra 32299. Simulation using DELPHINE (400 rays per antenna and call every $0.1 \mathrm{~s}$ ) in solid blue. Simulation using renormalized FEB profile in dash green.

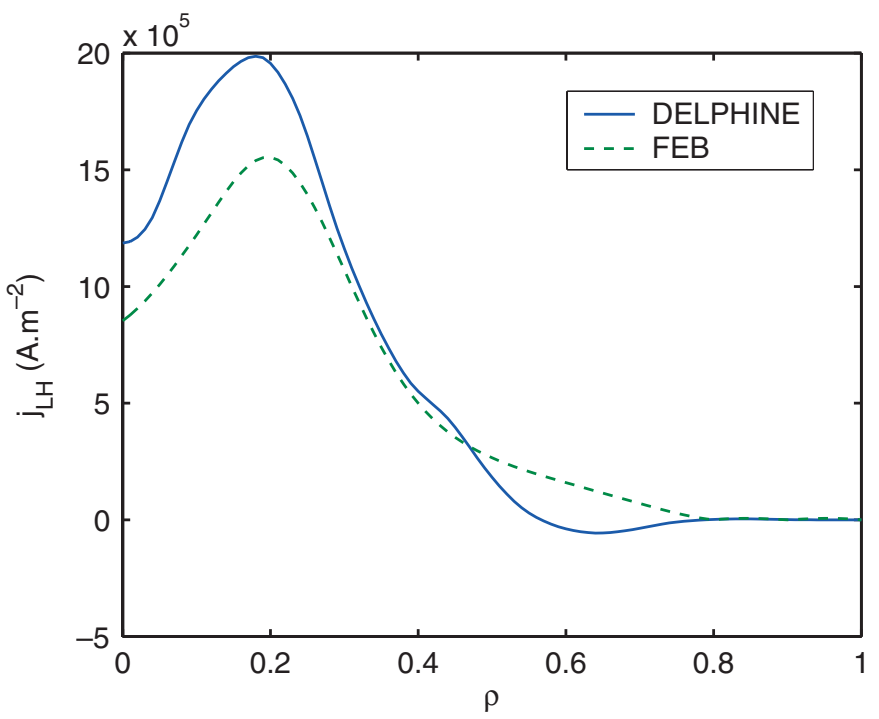

Figure 16. Current density profile in the steady-state phase of shot Tore Supra 32299. Simulation using DELPHINE (400 rays per antenna and call every $0.1 \mathrm{~s}$ ) in solid blue. Simulation using renormalized FEB profile in dash green

obtained if only the main peak around $n_{\| 0}=1.7$ is considered). Nevertheless, the off-axis negative current driven by the negative high $\left|n_{\|}\right|$components seems to be in contradiction to the real situation, since the CRONOS simulation using a positive current density around $\rho=0.65$ yields excellent agreement on $l_{\mathrm{i}}$. We cannot consider the neutralization of the negative off-axis current by the parallel electric field, which is zero in such a steady-state discharge with zero loop voltage. It might be that some mechanism broadens the $j_{\mathrm{LH}}$ profile by moving co-current driving electrons around $\rho=0.65$, which would balance the negative current at this point. 


\section{Discussion: capability of present LH modelling}

In this work we have illustrated the impact of the total current profile on the LH waves propagation and its consequences on the power deposition and driven current. The RT/FP method is able to grasp some qualitative features of this dependence, like the broadening of $j_{\mathrm{LH}}$ at high plasma current and the increase of current drive efficiency. The RT technique also shows a strong dependence of the LH waves propagation on the current profile shape. In the situation considered here (no accessibility constraints and moderate $T_{\mathrm{e}}<10 \mathrm{keV}$ which allows us to absorb the waves along most of the plasma radius), which is typical of present day L-mode discharges, the trend of LH waves is to balance the peakedness (resp. hollowness) of the total current profile by driving more hollow (resp. peaked) non-inductive current profile.

In discharges where the current profile is dominated by LHCD, this implies some autoregulation of $j$ and $j_{\mathrm{LH}}$ towards a medium peakedness. The auto-regulation mechanism should reduce the ability to control the LH power deposition by tuning the launched $n_{\|}$spectrum, though not completely hindering it [53]. However, this process makes the consistent current diffusion modelling of such discharges extremely challenging and demanding in computation time. Nevertheless, it has been possible to find a steady-state solution for an $80 \% \mathrm{LH}$ driven discharge, at the cost of one RT/FP calculation every $0.1 \mathrm{~s}$ of simulation time. The calculated LHCD profile was close to the experiment, though the measured FEB emission provides a more accurate determination of $j_{\mathrm{LH}}$.

Do we have any evidence of such a self-regulation process of $j$ and $j_{\mathrm{LH}}$ in experiments? Tore Supra has quite a large database of zero loop voltage shots dominated by LHCD, which provide some information relative to this issue. After the switch on of the LH power, the current profile of those shots evolves from its ohmic shape towards a new shape driven by fully noninductive means. On Tore Supra, this current profile evolution takes typically a few seconds, according to resistive current diffusion simulations. If the self-regulation process would play a significant role, then the FEB emission should evolve simultaneously with the plasma current profile, until this one has reached its final stationary state. In Tore Supra zero loop voltage experiments with dominant LHCD, the FEB profile is found to broaden during the LH power ramp-up (figure 17). This evolution might be related to the self-regulation mechanism but might also be the evidence of a correlation between LH power and its deposition profile width. Nevertheless, once the plateau LH power is reached, the FEB emission does not vary anymore, while the current profile is expected to continue to evolve during a few seconds (figure 17). Therefore it appears that the FEB emission profile is much less sensitive to the shape of the current profile than the LH driven current profile predicted by RT/FP calculations.

This is a general trend of the comparison between the experiment and the RT/FP results: the latter are usually quite sensitive to variations of input profiles (current density but also electron temperature, electron density), even when the number of rays is high enough to provide a relevant stochastic description of the wave propagation. Conversely, the measured FEB emission, which is the most direct representation of LHCD available, is generally less sensitive to these parameters. This suggests that some physics must be added to the classical RT/FP approach to account for the experimental phenomenology. There are several candidate broadening mechanisms, which may account for such a reduced sensitivity of the LH current profile with respect to the plasma profiles, at least qualitatively: radial transport of fast electrons [44,54], induced either by turbulence or by the LH waves themselves [55], scattering of the waves by density fluctuations $[8,13]$, broadening of the launched $n_{\|}$spectrum by parametric instabilities [56], wave diffraction [26,27] and breaking up of the quasilinear approximation [57]. Most of these effects are however very difficult to assess quantitatively by measurements. The only way to proceed further is to dedicate new efforts both to the modelling 


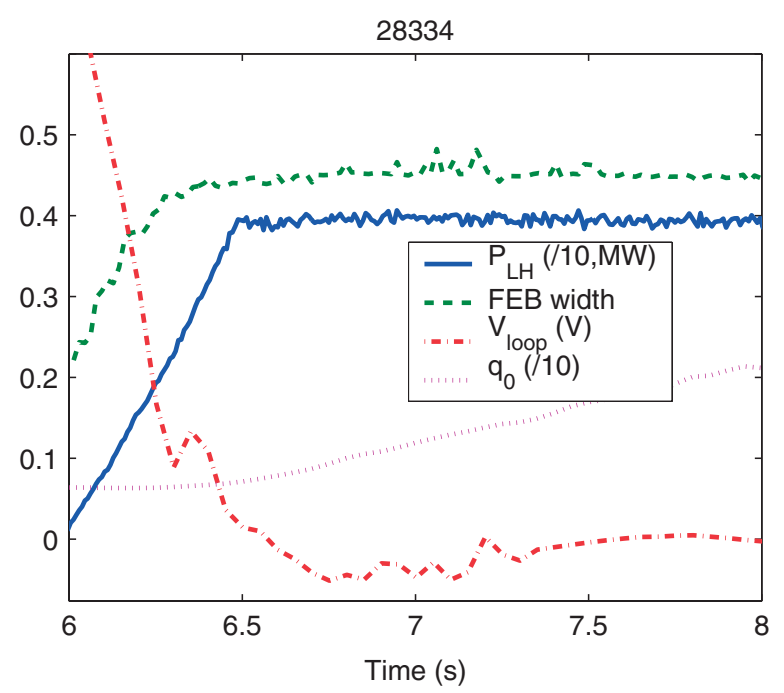

Figure 17. Time traces during the onset of LH power, shot 28334: LH power (solid, blue), width of the HXR inverted profile (dash, green), loop voltage (dash-dot, red), safety factor at the centre (dots, purple) from resistive current diffusion simulation.

of those complex phenomena and their careful benchmarking with a wide set of experiments. Until those developments are carried out, RT coupled to 2D in momentum space relativistic FP codes like DELPHINE remain the most precise tools available for LHCD predictions and have proved to account for at least some of the dependences observed during LHCD experiments.

\section{Appendix. Derivation of the equations for the Landau damping of LH waves on electrons}

The purpose of this appendix is to show in detail the derivation of equations (4) and (5) used by DELPHINE for the damping of LH waves on electrons. Though the method used is quite common, the detail of the calculations is often absent in the publications. Therefore it seems worthwhile to describe them thoroughly.

The Landau damping of LH waves is a kinetic effect since the resonance involves the velocity of the electrons. We have then to consider the hot plasma dielectric tensor, which is derived from a linearized treatment of the Vlasov equation and can be written in the general case as [58]

$$
\begin{gathered}
\bar{K}=\overline{1}+\frac{\omega_{\mathrm{p}}^{2}}{\omega^{2}} \int_{0}^{\infty} 2 \pi p_{\perp} \mathrm{d} p_{\perp} \int_{-\infty}^{\infty} \mathrm{d} p_{\|} \sum_{m=-\infty}^{\infty} \frac{\gamma^{-1} \bar{S}}{\left(\omega-k_{\|} v_{\|}-m \Omega_{c}\right)}, \\
\bar{S}=\left[\begin{array}{ccc}
v_{\perp} U\left(\frac{m J_{\mathrm{m}}}{x}\right)^{2} & \mathrm{i} v_{\perp} U \frac{m J_{\mathrm{m}} J_{\mathrm{m}}^{\prime}}{x} & v_{\perp} W \frac{m J_{\mathrm{m}}^{2}}{x} \\
-\mathrm{i} v_{\perp} U \frac{m J_{\mathrm{m}} J_{\mathrm{m}}^{\prime}}{x} & v_{\perp} U\left(J_{\mathrm{m}}^{\prime}\right)^{2} & -\mathrm{i} v_{\perp} W J_{\mathrm{m}} J_{\mathrm{m}}^{\prime} \\
v_{\|} U \frac{m J_{\mathrm{m}}^{2}}{x} & \mathrm{i} v_{\|} U J_{\mathrm{m}} J_{\mathrm{m}}^{\prime} & v_{\|} W J_{\mathrm{m}}^{2}
\end{array}\right],
\end{gathered}
$$


where

$$
\begin{aligned}
& U=M \omega \frac{\partial f}{\partial p_{\perp}}+k_{\|}\left(\frac{\partial f}{\partial p_{\|}} p_{\perp}-\frac{\partial f}{\partial p_{\perp}} p_{\|}\right), \\
& W=M \omega \frac{\partial f}{\partial p_{\|}}-M m \frac{\Omega_{c}}{p_{\perp}}\left(\frac{\partial f}{\partial p_{\|}} p_{\perp}-\frac{\partial f}{\partial p_{\perp}} p_{\|}\right) .
\end{aligned}
$$

$M$ is the relativistic mass $\left(M=m_{0} \gamma\right)$ and $x=k_{\perp} v_{\perp} / \Omega_{c}$. The argument of the Bessel functions $J_{\mathrm{m}}$ is $x$. $J_{\mathrm{m}}^{\prime}$ denotes the derivative of $J_{\mathrm{m}}$. The integer $m$ characterizes the harmonic of the possible cyclotron resonances. The electron distribution function $f$ is normalized as the following: $\int_{-\infty}^{\infty} \mathrm{d} p_{\|} \int_{0}^{\infty} 2 \pi p_{\perp} \mathrm{d} p_{\perp} f=1$, and $p_{\|}$and $p_{\perp}$ correspond respectively to the parallel and perpendicular components of the electron momentum with respect to the direction of the local magnetic field.

ELD is an $m=0$ resonance (Cerenkov condition $\omega-k_{\|} v_{\|}=0$ ), along the direction of the local magnetic field, i.e. corresponds to the $z z$ term of the dielectric tensor. At the resonance, the denominator of (A1) tends towards 0 , so it is useful to write the fraction as

$$
\frac{1}{\omega-k_{\|} v_{\|}}=P\left(\frac{1}{\omega-k_{\|} v_{\|}}\right)-\mathrm{i} \pi \delta\left(\omega-k_{\|} v_{\|}\right),
$$

where $\delta$ is the Dirac distribution and $P$ denotes the principal value (i.e. the value of the argument when it is finite, or 0 when $\left.\omega-k_{\|} v_{\|}=0\right)$. At the resonance, the $z z$ term of the dielectric tensor reduces then to

$$
K_{z z}=1-\mathrm{i} \pi \frac{\omega_{\mathrm{pe}}^{2}}{\omega^{2}} \int_{0}^{\infty} 2 \pi p_{\perp} \mathrm{d} p_{\perp} \int_{-\infty}^{\infty} \mathrm{d} p_{\|} m_{\mathrm{e}} v_{\|} J_{0}^{2} \omega \frac{\partial f}{\partial p_{\|}} \delta\left(\omega-k_{\|} v_{\|}\right) .
$$

Using $p_{\|}=\gamma m_{\mathrm{e}} v_{\|}$and integrating over $p_{\|}$

$$
K_{z z}=1-\left.\mathrm{i} \pi \operatorname{sgn}\left(k_{\|}\right) \frac{\omega_{\mathrm{p}}^{2}}{k_{\|}^{2}} \int_{0}^{\infty} 2 \pi p_{\perp} \mathrm{d} p_{\perp} \gamma m_{\mathrm{e}}^{2} J_{0}^{2} \frac{\partial f}{\partial p_{\|}}\right|_{p_{\|}=\gamma m_{\mathrm{e}}\left(\omega / k_{\|}\right)},
$$

where $\gamma$ is evaluated at the resonance.

In the quasilinear theory, the damping phenomena correspond to the antihermitian terms of the dielectric tensor [10], which for ELD is the imaginary part of $K_{z z}$. The damping rate along the rays is calculated by evaluating the impact of the imaginary part of $K_{z z}$ on the dispersion relation. We recall here that everywhere in the plasma, the electric field of the wave must satisfy the equation [59]

$$
\vec{k} \times(\vec{k} \times \vec{E})+\left(\frac{\omega}{c}\right)^{2} \bar{K} \cdot \vec{E}=\overrightarrow{0}
$$

which can be written as $\vec{M} \cdot \vec{E}=\overrightarrow{0}$, with

$$
\bar{M}=\left[\begin{array}{ccc}
\varepsilon_{\perp}-n_{\|}^{2} & -\mathrm{i} \varepsilon_{x y} & n_{\|} n_{\perp} \\
\mathrm{i} \varepsilon_{x y} & \varepsilon_{\perp}-n^{2} & 0 \\
n_{\|} n_{\perp} & 0 & K_{z z}-n_{\perp}^{2}
\end{array}\right],
$$

where all elements of the dielectric tensor but $K_{z z}$ have been written in the cold plasma approximation, since we consider only the kinetic effects corresponding to Landau damping on electrons. The plasma dispersion relation is obtained by writing that equation (A6) should admit a nontrivial solution, i.e. the determinant of $\bar{M}$ should be zero. Therefore the imaginary part of $K_{z z}$ introduces an imaginary part in the dispersion relation $D$, which can be written as

$$
\operatorname{Im}(D)=\operatorname{Im}\left(K_{z z}\right)\left[\left(\varepsilon_{\perp}-n_{\|}^{2}\right)\left(\varepsilon_{\perp}-n^{2}\right)-\varepsilon_{x y}^{2}\right] .
$$


In order to fulfil the dispersion relation $D=0$, the wave frequency should also have an imaginary part $\omega=\omega_{\mathrm{r}}-\mathrm{i} \gamma_{\mathrm{abs}}$; therefore $\gamma_{\mathrm{abs}}$ defines the damping rate of the LH wave electric field. By linearizing $D\left(\omega_{\mathrm{r}}-\mathrm{i} \gamma_{\mathrm{abs}}\right)=0$ in the weak absorption limit $\left(\gamma_{\mathrm{abs}} \ll \omega_{\mathrm{r}}\right)$, we obtain

$$
\gamma_{\mathrm{abs}}=\frac{\operatorname{Im}\left(D\left(\omega_{\mathrm{r}}\right)\right)}{\partial \operatorname{Re}\left(D\left(\omega_{\mathrm{r}}\right)\right) / \partial \omega} .
$$

Therefore we obtain

$$
\begin{aligned}
\gamma_{\mathrm{abs}}=\frac{\left[\left(\varepsilon_{\perp}-n_{\|}^{2}\right)\left(\varepsilon_{\perp}-n^{2}\right)-\varepsilon_{x y}^{2}\right]}{\partial \operatorname{Re}\left(D\left(\omega_{\mathrm{r}}\right)\right) / \partial \omega} \\
\quad \times\left(-\left.\pi \operatorname{sgn}\left(k_{\|}\right) \frac{\omega_{\mathrm{pe}}^{2}}{k_{\|}^{2}} \int_{0}^{\infty} 2 \pi p_{\perp} \mathrm{d} p_{\perp} m_{\mathrm{e}}^{2} J_{0}^{2} \gamma \frac{\partial f}{\partial p_{\|}}\right|_{p_{\|}=\gamma m_{\mathrm{e}}\left(\omega / k_{\|}\right)}\right) .
\end{aligned}
$$

As discussed in the main text (section 2), in typical Tore Supra cases, the dominant contribution to the integral occurs for small values of $p_{\perp}$. Therefore we evaluate the expression under the integral in the limit $p_{\perp} \approx 0$, which yields $J_{0}^{2} \approx 1$, and $\gamma$ is evaluated at the resonance as $\gamma \approx 1 \sqrt{1-\left(1 / n_{\|}^{2}\right)}$. Introducing $f_{\|}=\int_{0}^{\infty} 2 \pi p_{\perp} \mathrm{d} p_{\perp} f$, we obtain the expression (4) used to calculate the damping in the DELPHINE code:

$\gamma_{\mathrm{abs}}=\frac{\left[\left(\varepsilon_{\perp}-n_{\|}^{2}\right)\left(\varepsilon_{\perp}-n^{2}\right)-\varepsilon_{x y}^{2}\right]}{\partial \operatorname{Re}\left(D\left(\omega_{\mathrm{r}}\right)\right) / \partial \omega}\left(-\left.\pi \operatorname{sgn}\left(k_{\|}\right) \frac{\omega_{\mathrm{pe}}^{2}}{k_{\|}^{2}} \gamma m_{\mathrm{e}}^{2} \frac{\partial f_{\|}}{\partial p_{\|}}\right|_{p_{\|}=\gamma m_{\mathrm{e}}\left(\omega / k_{\|}\right)}\right)$.

The energy being proportional to the square of the WKB electric field $\vec{E}=\vec{E}_{0} \mathrm{e}^{\mathrm{i}(\vec{k}, \vec{r}-\omega t)}$, the power $P$ carried along the ray is damped following

$$
\frac{\mathrm{d} P}{\mathrm{~d} t}=-2 \gamma_{\mathrm{abs}} P \text {. }
$$

In order to calculate the electron distribution function self-consistently with the power absorption along the rays, it is useful to derive the expression of the quasilinear diffusion coefficient which appears in the FP equation. An incremental quasilinear diffusion coefficient $\Delta D_{\mathrm{QL}}$ is associated with each time step $\Delta t$ along the ray path. In the momentum space, the electron flux along the parallel direction associated with this quasilinear diffusion coefficient is equal to

$$
-n_{\mathrm{e}} V \int_{0}^{\infty} 2 \pi p_{\perp} \mathrm{d} p_{\perp} \Delta D_{\mathrm{QL}} \frac{\partial f}{\partial p_{\|}}
$$

where the density $n_{\mathrm{e}}$ has been multiplied by the volume of the real space $V$, so that this flux represents a number of electron diffusing through the point $p_{\|}$per unit of time. Hence the power associated with the quasilinear diffusion of electrons between $p_{\|}$and $p_{\|}+\mathrm{d} p_{\|}$is

$$
\begin{aligned}
\left.\mathrm{d} P\right|_{p_{\|}} ^{p_{\|}+\mathrm{d} p_{\|}} & =-n_{\mathrm{e}} V \int_{0}^{\infty} 2 \pi p_{\perp} \mathrm{d} p_{\perp} \Delta D_{\mathrm{QL}} \frac{\partial f}{\partial p_{\|}} \mathrm{d}\left(\gamma m_{\mathrm{e}} c^{2}\right) \\
& =-n_{\mathrm{e}} V \int_{0}^{\infty} 2 \pi p_{\perp} \mathrm{d} p_{\perp} \Delta D_{\mathrm{QL}} \frac{\partial f}{\partial p_{\|}} \frac{p_{\|}}{\gamma m_{\mathrm{e}}} \mathrm{d} p_{\|} .
\end{aligned}
$$

From the RT point of view, the power damped during a progress in time $\Delta t$ along the ray path is equal to $2 \gamma_{\mathrm{abs}} P \Delta t$. Using the expression of $\gamma_{\mathrm{abs}}$ prior to the integration over $p_{\|}$(equation (A4)), this yields the power absorbed by electrons whose parallel momentum lies between $p_{\|}$and $p_{\|}+\mathrm{d} p_{\|}$as

$$
\begin{array}{rl}
\left.\mathrm{d} P\right|_{p_{\|}} ^{p_{\|}+\mathrm{d} p_{\|}}=2 & P \Delta t \frac{\left[\left(\varepsilon_{\perp}-n_{\|}^{2}\right)\left(\varepsilon_{\perp}-n^{2}\right)-\varepsilon_{x y}^{2}\right]}{\partial D / \partial \omega} \\
& \times\left(-\pi \frac{\omega_{\mathrm{pe}}^{2}}{\omega} m_{\mathrm{e}} p_{\|} \frac{1}{\left|k_{\|}\right|} \int_{0}^{\infty} 2 \pi p_{\perp} \mathrm{d} p_{\perp} J_{0}^{2} \frac{\partial f}{\partial p_{\|}} \delta\left(p_{\|}-\gamma m_{\mathrm{e}} \frac{\omega}{k_{\|}}\right)\right) \mathrm{d} p_{\|} .
\end{array}
$$


The comparison of (A14) and (A15) provides the expression of the quasilinear diffusion coefficient as

$\Delta D_{\mathrm{QL}}=\frac{2 P \Delta t}{n_{\mathrm{e}} V} \frac{\left[\left(\varepsilon_{\perp}-n_{\|}^{2}\right)\left(\varepsilon_{\perp}-n^{2}\right)-\varepsilon_{x y}^{2}\right]}{\partial D / \partial \omega}\left(\pi \frac{\omega_{\mathrm{pe}}^{2}}{\omega} \gamma m_{\mathrm{e}}^{2} J_{0}^{2} \frac{1}{\left|k_{\|}\right|} \delta\left(p_{\|}-\gamma m_{\mathrm{e}} \frac{\omega}{k_{\|}}\right)\right)$

which is the expression used in the DELPHINE code.

\section{References}

[1] Jacquinot J 2003 Nucl. Fusion 431583

[2] Zushi H et al 2003 Nucl. Fusion 431600

[3] Litaudon X et al 2002 Plasma Phys. Control. Fusion 441057

[4] Challis C D et al 2001 Plasma Phys. Control. Fusion 43861

[5] Equipe Tore Supra 1996 Plasma Phys. Control. Fusion 38 A251

[6] Litaudon X et al 2001 Plasma Phys. Control. Fusion 43677

[7] Taylor T S 1997 Plasma Phys. Control. Fusion 39 B47

[8] Bonoli P T and Ott E 1982 Phys. Fluids 25359

[9] Bonoli P T and Englade R C 1986 Phys. Fluids 292937

[10] Brambilla M and Cardinali A 1982 Plasma Phys. 241187

[11] Bizarro J P and Moreau D 1993 Phys. Fluids B 51227

[12] Fuchs V et al 1995 Nucl. Fusion 351

[13] Baranov Y et al 1996 Nucl. Fusion 361031

[14] Esterkin A R and Piliya A D 1996 Nucl. Fusion 361501

[15] Peysson Y et al 1996 Phys. Plasmas 33668

[16] Barbato E and Romanelli F 1990 Phys. Fluids B 22687

[17] Fisch N J 1982 Rev. Mod. Phys. 59175

[18] Peysson Y and the Tore Supra team 2000 Plasma Phys. Control. Fusion 42 B87

[19] Peysson Y et al 1998 Proc. 17th IAEA Fusion Energy Conf. (Yokohama, Japan) IAEA-CN-69/CDP/03

[20] Peysson Y 1999 Proc. 13th Top. Conf. on Radio Frequency Power in Plasmas (Annapolis, USA) vol 485, ed S Bernabei and F Paoletti p 183

[21] Peysson Y and Imbeaux F 1999 Rev. Sci. Instrum. 703987

[22] Ekedahl A et al 1998 Nucl. Fusion 381397

[23] Barbato E et al 2004 Fusion Sci. Technol. 45323

[24] Press W H, Teukolsky S A, Vetterling W T and Flannery B P 1992 Numerical Recipes in C 2nd edn (Cambridge: Cambridge University Press)

[25] Brambilla M 1998 Kinetic Theory of Plasma Waves (Oxford: Oxford Science Publications)

[26] Pereverzev G V 1998 Phys. Plasmas 53529

[27] Poli E, Pereverzev G V and Peeters A G 1999 Phys. Plasmas 65

[28] Moreau D, Rax J M and Samain A 1989 Plasma Phys. Control. Fusion 311895

[29] Peysson Y et al 1998 Nucl. Fusion 38939

[30] Killeen J et al 1986 Computational Methods for Kinetic Models of Magnetically Confined Plasmas (Berlin: Springer)

[31] Braams B J and Karney C F F 1989 Phys. Fluids B 11355

[32] Peysson Y and Shoucri M 1998 Comput. Phys. Commun. 10955

[33] Decker J and Peysson Y 2004 Report EUR-CEA-FC-1736

[34] Inc. The MathWorks Inc. 2002 Matlab, the Language of Technical Computing, Version 6 (Natick, USA: The MathWorks Inc.)

[35] G T A Huysmans et al 1991 CP90: Conf. on Comp. Physics (Amsterdam, The Netherlands) (Singapore: World Scientific) p 371

[36] Kupfer K, Moreau D and Litaudon X 1993 Phys. Fluids B 54391

[37] Bonoli P T 1984 IEEE Trans. Plasma Sci. 1295

[38] Paoletti F et al 1994 Nucl. Fusion 34771

[39] Peysson Y and the Tore Supra team 2001 Nucl. Fusion 411703

[40] Takase H, Okano K and Hatayama A 1991 Plasma Phys. Control. Fusion 33749

[41] Ushigusa K 1996 Plasma Phys. Control. Fusion 381825 
[42] Beaumont B et al 2001 Fusion Eng. Des. 56-57 667

[43] Pereverzev G et al 1991 Report IPP 5/42

[44] Peysson Y 1993 Plasma Phys. Control. Fusion 35 B253

[45] Imbeaux F 1999 Report EUR-CEA-FC-1679

[46] Hirshman S P 1988 Phys. Fluids 313150

[47] Giruzzi G et al 2003 Phys. Rev. Lett. 91135001

[48] Imbeaux F et al 2004 Proc. 20th IAEA Fusion Energy Conf. (Vilamoura, Portugal) IAEA-CN-116/EX/P6-16

[49] Tala T J J et al 2000 Nucl. Fusion 401635

[50] Basiuk V et al 2003 Nucl. Fusion 43822

[51] Van Houtte D et al 2004 Nucl. Fusion 44 L11

[52] Moreau D and Nguyen T K 1984 Report EUR-CEA-FC-1246

[53] Imbeaux F and Peysson Y 1999 Proc. 26th EPS Conf. on Control. Fusion and Plasma Phys. (Maastricht) p 1017

[54] Giruzzi G et al 1995 Phys. Rev. Lett. 74550

[55] Kupfer K and Bers A 1991 Phys. Fluids B 32783

[56] Cesario R et al 2004 Phys. Rev. Lett. 92175002

[57] Pavlo P and Krlin L 1999 Plasma Phys. Control. Fusion 41541

[58] Bekefi G 1966 Radiation Processes in Plasmas (New York: Wiley)

[59] Stix T H 1962 The Theory of Plasma Waves (New York: McGraw-Hill) 